\title{
A Functional Integral Approach to Shock Wave Solutions of Euler Equations with Spherical Symmetry
}

\author{
Tong Yang \\ School of Mathematics, Institute for Advanced Study, Princeton, NJ 08540, USA
}

Received: 7 March 1994

\begin{abstract}
For $n \times n$ systems of conservation laws in one dimension without source terms, the existence of global weak solutions was proved by Glimm [1]. Glimm constructed approximate solutions using a difference scheme by solving a class of Riemann problems.

In this paper, we consider the Cauchy problem for the Euler equations in the spherically symmetric case when the initial data are small perturbations of the trivial solution, i.e., $u \equiv 0$ and $\rho \equiv$ constant, where $u$ is velocity and $\rho$ is density. We show that this Cauchy problem can be reduced to an ideal nonlinear problem approximately. If we assume all the waves move at constant speeds in the ideal problem, by using Glimm's scheme and an integral approach to sum the contributions of the reflected waves that correspond to each path through the solution, we get uniform bounds on the $L_{\infty}$ norm and total variational norm of the solutions for all time. The geometric effects of spherical symmetry leads to a non-integrable source term in the Euler equations. Correspondingly, we consider an infinite reflection problem and solve it by considering the cancellations between reflections of different orders in our ideal problem. Thus we view this as an analysis of the interaction effects at the quadratic level in a nonlinear model problem for the Euler equations. Although it is far more difficult to obtain estimates in the exact solutions of the Euler equations due to the problem of controlling the time at which the cancellations occur, we believe that this analysis of the wave behaviour will be the first step in solving the problem of existence of global weak solutions for the spherically symmetric Euler equations outside of fixed ball.
\end{abstract}

\section{Introduction}

We consider the Euler equations of the compressible gas dynamics in $R^{n}$,

$$
\begin{aligned}
& \rho_{t}+\nabla \cdot(\rho \vec{u})=0, \\
& (\rho \vec{u})_{t}+\nabla \cdot(\rho \vec{u} \otimes \vec{u}+P)=0,
\end{aligned}
$$


here $t \in R, x \in R^{n}, \rho$ is the density, $\vec{u}$ is the velocity, and $P=P(\rho)$ is the pressure. In particular, this models an isothermal gas, i.e., $P(\rho)=\rho$ which can be viewed as a model problem obtained by linearizing the pressure. We consider here spherically symmetric solutions of (1) in three dimensions outside a ball of radius 1 . As is well known, Glimm's method [1] applies in the case $n=1([9,10,11])$, but when $n>1$, there is no general existence theory for weak (shock wave) solutions of (1) and there is only short time existence of classical solutions $([2,3])$. Recently, the authors in [4] proved that there exists a linear growth rate for a system of spherically symmetric solutions in Lagrangian coordinates. In the spherically symmetric case, Eqs. (1) can be reduced to one dimensional equations in the radial variable $x$, but there is a non-integrable source term of order $1 / x$. As a result, it is an open problem to show that spherically symmetric solutions of (1) remain bounded for all time. It was observed in [12] that waves moving out to infinity will generate an infinite amount of wave strength due to the reflection of waves by the non-integrable source, and this occurs when the strength is measured in the norm that preserves the outgoing and incoming wave strengths to the leading order with respect to the amplitude of the perturbation. (The explicit definition of reflection will be given in Sect. 6.)

In this paper we show that the above problem can be reduced to an ideal nonlinear problem approximately. To overcome the geometric effects in the Euler equations, we attempt the resolve an infinite reflection problem by taking a functional integral approach to summing the strengths of the reflected waves in the ideal problem. The idea is to reconstruct wave strengths at time $t>0$ from the wave strengths at time zero by summing the contributions of the reflected waves that correspond to each path through the solution. In this way we can account for the "sign" of reflected waves, and thus take account of cancellations that occur due to mutiple reflections of waves. This procedure is analogous to the Feynman path integral approach to quantum mechanics. Our result is that, assuming that the wave speeds are constants, the total wave strengths remain uniformly bounded for all time in the solutions of the ideal problem. Although this is just a model for wave propagation in spherically symmetric solutions of (1), this analysis resolves the non-summability problem observed in [12] in the simplest setting where it occurs. Moreover, we believe that this gives a quantitative explanation for why the total wave strengths remain bounded for all time in the presence of a non-integrable source term. In particular, when the wave speeds are allowed to be nonlinear, it is difficult to obtain sharp estimates for the net cancellation of waves due to mutiple reflections because of the problem of controlling the time at which the cancellations occur. Nevertheless, although it is far more difficult to obtain estimates in the exact problem, we suggest that the underlying mechanism that bounds the wave strengths in the full nonlinear problem is essentially the same as in the model problem considered here. Our analysis leads to an interesting new norm for the initial data in which the total variation of the approximate solutions is bounded by the initial data in this norm.

Throughout this paper, we consider the case when $n=3$. How to prove a similar result for $n \neq 3$ is still an open problem. But the 3-dimensional case is interesting by itself because we know that Huygen's principle holds for wave equations in three dimensions. Furthermore, it is pointed out in [13] that $n=3$ is a critical value when we consider the asymptotic behaviour as time tends to infinity. That is, the nonlinear effects will dominate the geometric ones for $n<3$ and shocks form at algebraic rates in asymptotic analysis. But for $n>3$, the geometric effects are so strong that they will smooth out the nonlinear sigularities in the asymptotic states. While at 
the critical value $n=3$, shocks form at an exponential rate asymptotically, Another reason why we choose $n=3$ is that when we calculate the reflected wave, there is a factor $\frac{n-1}{2}$ in the strength of the reflected wave. Thus the factor becomes $\left(\frac{n-1}{2}\right)^{l}$ for an $i$-times reflected wave. Since we are going to calculate the cancellation between $i$-times reflected waves and those reflected $i+2$ times, it is easier to let $\frac{n-1}{2}=1$, i.e. $n=3$.

Under the assumption of spherical symmetry, Eqs. (1) can be rewritten as

$$
\begin{aligned}
& (a \rho)_{t}+(a \rho u)_{x}=0, \\
& (a \rho u)_{t}+\left(a \rho u^{2}+a p\right)_{x}=a^{\prime} p, \quad x \geqq 1, t>1,
\end{aligned}
$$

with initial and boundary data

$$
\begin{aligned}
& u(x, 0)=u_{0}(x), \quad \rho(x, 0)=\rho_{0}(x), \quad x \geqq 1, \\
& u(1, t)=0, \quad t \geqq 0,
\end{aligned}
$$

where $a=x^{2}, x \geqq 1$.

We linearize the pressure by assuming $p=\sigma^{2} \rho$. Without loss of generality, we assume the sound speed $\sigma=1$. If $a=$ constant, (2) can be rewritten as

$$
\begin{aligned}
& \rho_{t}+(\rho u)_{x}=0, \\
& (\rho u)_{t}+\left(\rho u^{2}+\rho\right)_{x}=0,
\end{aligned}
$$

and for smooth solutions, (2) be rewritten as

or

$$
\begin{aligned}
(a \rho)_{t}+(a \rho u)_{x} & =0, \\
u_{t}+\left(\frac{u^{2}}{2}+\ln \rho\right)_{x} & =0,
\end{aligned}
$$

$$
\begin{aligned}
& \rho_{t}+(\rho u)_{x}=-\frac{2 \rho u}{x}, \\
& u_{t}+\left(\frac{u^{2}}{2}+\ln \rho\right)_{x}=0 .
\end{aligned}
$$

By (5), we know that the strength of the source is of order $1 / x$, and the $L_{1}$ norm of this strength is infinite as $x$ tends to infinity. Thus the method used in $[5,6]$ does not apply to this case.

Remark 1. By splitting variables, Eqs. (5) imply some kind of exponential growth locally for $\rho$. Actually, we can prove that there exist a lower bound $C T^{\ln T}$ and an upper bound $C e^{T}$ for the total reflections at time $T$, if we do not consider the cancellations between them, where $C$ is a constant independent of $T$. Thus we believe that because of the boundary conditions we choose, the uniform bound for the sum of the total reflections is a global property, and this problem can not be localized.

We will show that the Cauchy problem (2) and (3) can be reduced to an ideal nonlinear problem approximately. To do this, we will solve the following generalized 
Riemann problems, i.e., Eqs. (2) with initial data

$$
(u, \rho, a)= \begin{cases}\left(u_{l}, \rho_{l}, a_{l}\right), & x<0, \\ \left(u_{r}, \rho_{r}, a_{r}\right), & x>0,\end{cases}
$$

where $\rho_{i}, u_{i}$ and $a_{i} i=l, r$ are constants. It is straightforward to check that when $a=$ constant, Eqs. (2) have two eigenvalues $\lambda_{i}= \pm 1+u$ with corresponding eigenvectors $\mathscr{R}_{l}=\left(\left(1+\lambda_{i}^{2}\right)^{-\frac{1}{2}}, \lambda_{i}\left(1+\lambda_{i}^{2}\right)^{-\frac{1}{2}}\right), i=1,2$ respectively. Thus for small $|u|$, (2) is strictly hyperbolic and genuinely nonlinear in the sense of Lax, i.e., $\lambda_{1}<\lambda_{2}$ and $\mathscr{R}_{i} \cdot \nabla \lambda_{i} \neq 0, i=1,2$ respectively. It is well known that there exist two families of hyperbolic waves in the solutions of the Riemann problems. From here on, we call the waves corresponding to $\lambda_{i}$ as hyperbolic waves of the $i^{\text {th }}$ family.

The solutions to the Riemann problem (2) and (6) will be constructed in Sect. 4. In the construction, we modify $a(x)$ by a family of step functions as a duct modified by discontinuous diameters. To resolve the jump of $a(x)$ in the Riemann problem at $x=0$, we need following definitions.

Definition 1. We define a standing wave at $x=0$ with strength $\left|u_{r}-u_{l}\right|$ to be $a$ solution of the Riemann problems (2) and (6), satisfying

$$
\begin{gathered}
(a \rho u)_{x}=0 \\
\left(\frac{u^{2}}{2}+\ln \rho\right)_{x}=0,
\end{gathered}
$$

in the weak sense, i.e.,

$$
\begin{aligned}
a_{l} \rho_{l} u_{l} & =a_{r} \rho_{r} u_{r}, \\
\frac{u_{1}^{2}}{2}+\ln \rho_{l} & =\frac{u_{r}^{2}}{2}+\ln \rho_{r} .
\end{aligned}
$$

As shown in [7], we can solve the Riemann problem by hyperbolic waves on $x<0$ and $x>0$, and standing waves at $x=0$. Since we assume $|u|$ is small, it was proved in [7] that the solution is unique.

Throughout this paper, we use a new norm to measure hyperbolic waves. It was shown in [12] that this norm preserves the strengths of the outgoing and incoming waves to the leading term with respect to the amplitude of the perturbation.

Definition 2. For any hyperbolic wave $\gamma$ sitting on $a(x)=x^{2}$ with left state $\left(u_{l}, \rho_{l}\right)$ and right state $\left(u_{r}, \rho_{r}\right)$, we define the norm of $\gamma$ by

$$
\|\gamma\|=\left|x\left(u_{r}-u_{l}\right)\right|
$$

while for any wave, Glimm's strength is defined by

$$
|\gamma|=\left|u_{r}-u_{l}\right|
$$

In this paper, we consider a model problem for (2) and (3) in which the $1^{\text {st }}$ and $2^{\text {nd }}$ family of hyperbolic waves move at $-\xi$ and $\xi$ respectively, where $\xi$ is a positive constant. We introduce the following norm:

$$
\||\phi|\| \equiv\left\|\frac{1}{x}\left\{\operatorname{Var}_{[x, \infty)}\{y \phi(y)\}\right\}\right\|_{L_{1}(1, \infty)},
$$


for any function $\phi(x)$ for which $\|\phi\| \|$ is defined, where the variation is taken in the interval $[x, \infty)$.

Now we can state the main theorem in this paper.

Theorem 1. For our model problem, there exist positive constants $\bar{\rho}$ and $\delta$ such that for $x \geqq 1$, if

$$
\begin{gathered}
\left|\rho_{0}(x)-\bar{\rho}\right|<\delta, \quad \operatorname{Var}\left\{\rho_{0}(x)\right\}<\delta, \\
\left|x u_{0}(x)\right|<\delta, \quad \operatorname{Var}\left\{x u_{0}(x)\right\}<\delta, \\
||\left|u _ { o } ( x ) \left\|\left|<\delta, \quad\left\|\rho_{o}(x)\right\|\right|<\delta,\right.\right. \\
\left\|u_{0}(x) \ln x\right\|_{L_{1}(1, \infty)}<\delta,
\end{gathered}
$$

where $\delta$ is sufficiently small, such that within the leading term of $\delta$ we have

$$
\begin{gathered}
|\rho(x, t)-\bar{\rho}|<G \delta, \quad|x u(x, t)|<G \delta, \\
\operatorname{Var}\{\rho(\cdot, t)\}, \operatorname{Var}\{u(\cdot, t)\}<G \delta,
\end{gathered}
$$

for all $x \geqq 1$ and $t \geqq 0$, where $G$ is a positive constant independent of time.

Remark 2. Actually, we can assume the hyperbolic waves of the $1^{\text {st }}$ family move at speed $-\xi_{1}$ and those of the $2^{\text {nd }}$ family at $\xi_{2}$, where $\xi_{1}$ and $\xi_{2}$ are positive constants. If $\left|\xi_{1}-\xi_{2}\right|=O(\delta)$ and the random sequence $\left\{a_{j}\right\}_{j=1}^{\infty}$ is equidistributed, then we can follow the proof in this paper and get the same result as Theorem 1 .

Remark 3. The conditions (A) and (B) are reasonable in the following sense. If there exist only standing waves initially in the difference scheme for $x>1$, then within the leading term $u_{0}(x)=\frac{c}{x^{2}}$ for some constant $c>0, x>1$. The condition (A) and (B) are obviously true in this case when $c$ is small. For sufficiently small $\delta$, condition (A) and (B) give a small perturbation of this case.

Now we consider a case when there exists only a hyperbolic wave $\gamma$ of the $1^{\text {st }}$ family emitting from $\left(x_{0}, 0\right)$. As shown in [12], the total norm of the reflected waves of $\gamma$ created by crossing the standing waves is $\|\gamma\| \ln x_{0}$ if time is large enough. This family of waves will be defined as the $1^{\text {st }}$ reflection of $\gamma$ in Sect. 7. In this case, the initial data $u_{0}(x)$ can be chosen as

$$
u(x, 0)= \begin{cases}0, & 1 \leqq x \leqq x_{0}, \\ \frac{c x_{0}^{2}}{x^{2}}, & x>x_{0},\end{cases}
$$

for some constant $c>0$. We have

$$
\begin{aligned}
\left\|u_{0}(x)\right\| \| & >\int_{1}^{x_{0}} \frac{1}{x}\left(\frac{c_{2} x_{0}^{2}}{x_{0}}\right) d x \\
& =x_{0} c_{2} \ln x_{0} .
\end{aligned}
$$

Since $\|\gamma\|=O\left(x_{0} c_{2}\right)$, then by condition (A) and (B), we have

$$
\|\gamma\| \ln x_{0} \leqq O(\delta) \text {. }
$$


Note 1. Throughout this paper, we use $O(b)$ to denote $|O(b)| \leqq K b$, where $K$ is a positive constant independent of $N, m, i$ and $\Delta_{x}$ which will appear later.

Thus under condition (A) and (B), we can conclude that the first reflection of a hyperbolic wave of the $1^{\text {st }}$ family is bounded in the norm defined in Definition 2 . As we will see later, the higher order reflections are bounded due to the cancellations between reflections of different orders.

\section{Definition of the Ideal Problem}

In this section, we define an ideal nonlinear problem. And we will prove that the Cauchy problem (2) and (3) can be reduced to it approximately in Sect. 5.

The ideal problem is an implicit hyperbolic system

$$
P(a(x), u(x, t), \rho(x, t))=0, \quad x \geqq 1, t \geqq 0
$$

Any Riemann problem with $(u, \rho)$ components of the left and right states lying in a small neighborhood of a constant state $\left(u_{0}, \rho_{0}\right)$ can be solved uniquely. The solution consists of three families of waves: the $1^{\text {st }}$ family moves at negative speed, while the $3^{\text {rd }}$ family moves at positive speed, and the $2^{\text {nd }}$ family moves at zero speed. As usual, the waves of the $1^{\text {st }}$ and $3^{\text {rd }}$ families are called as hyperbolic waves, i.e. rarefaction waves and shocks; and the waves of the $2^{\text {nd }}$ family are called as standing waves. For a hyperbolic wave, we define a norm to measure its strength, denoted by $\|\cdot\|$.

The waves interaction satisfies the following conditions. We use $\gamma^{1}\left(\gamma^{2}\right)$ to denote the waves of the $1^{\text {st }}\left(3^{\text {rd }}\right)$ family and $\gamma^{0}$ for the $2^{\text {nd }}$ family, and use

$$
\gamma^{2}+\gamma^{0}+\gamma^{1} \rightarrow \bar{\gamma}^{-1}+\bar{\gamma}^{0}+\bar{\gamma}^{2}
$$

to denote that the interaction of $\gamma^{i} \mathrm{~s}, i=0,1,2$, will create three new waves $\bar{\gamma}_{i} \mathrm{~s}$, $i=0,1,2$. Then for a standing wave $\gamma^{0}$ with a component of the left and right states being $a\left(x_{1}\right)$ and $a\left(x_{r}\right)$, we have

$$
\begin{aligned}
& \gamma^{0}+\gamma^{1} \rightarrow \bar{\gamma}^{1}+\bar{\gamma}^{0}+\bar{\gamma}^{2}, \\
& \left\|\bar{\gamma}^{1}\right\|=\left\|\gamma^{1}\right\|, \quad\left\|\bar{\gamma}^{2}\right\|=\frac{x_{r}-x_{l}}{x_{l}}\left\|\gamma^{1}\right\| .
\end{aligned}
$$

Furthermore, if $\gamma^{1}$ is a rarefaction wave (shock), then $\bar{\gamma}^{-1}$ and $\bar{\gamma}^{-2}$ are rarefaction waves (shocks).

$$
\begin{aligned}
& \gamma^{2}+\gamma^{0} \rightarrow \bar{\gamma}^{-1}+\bar{\gamma}^{0}+\bar{\gamma}^{2} \\
& \left\|\bar{\gamma}^{2}\right\|=\left\|\gamma^{2}\right\|, \quad\left\|\bar{\gamma}^{-1}\right\|=\frac{x_{r}-x_{l}}{x_{l}}\left\|\gamma^{2}\right\| .
\end{aligned}
$$

Furthermore, if $\gamma^{2}$ is a rarefaction wave (shock), then $\bar{\gamma}^{2}$ is a rarefaction wave (shock) and $\bar{\gamma}^{-1}$ is a shock (rarefaction wave).

- Hyperbolic waves of different families can cross each other with their norms and families unchanged. 
- For any hyperbolic wave $\gamma^{1}$ of the $1^{\text {st }}$ family impinging on the boundary $x=1$, the rebounded wave $\gamma^{2}$ is of $2^{\text {nd }}$ family which is shock (rarefaction wave) if $\gamma^{1}$ is shock (rarefaction wave), and

$$
\left\|\gamma^{2}\right\|=\left\|\gamma^{1}\right\| \text {. }
$$

Remark 4. In this paper, we choose a "solid wall" boundary condition on $x=1$. Under this condition, we have to consider the cancellation between reflected waves of a same original wave. We may choose other boundary conditions, but the analysis here applies only to the one we choose.

Actually, we will solve such an ideal problem corresponding to the model problem in Theorem 1. That is, we solve a model problem for the Cauchy problem (2) and (3).

\section{Derivation of Equations (2)}

In this section, we will derive Eqs. (2) in two physical models: The first model is a compressible fluid flow in a variable duct, and the second is a fluid flow in spherically symmetric gas dynamics. As pointed out in [12], Eqs. (2) are approximate conservation laws of mass and momentum for fluid flows in a variable area duct with the area of the cross section being $a(x)$, which satisfies $\left|a^{\prime}(x)\right| \ll 1$. And they are exact conservation laws of mass and momentum for spherically symmetric flow in $R^{3}$

The following derivation is borrowed from [12].

Firstly, we consider fluid flow in a variable area duct. In particular, we assume the boundary of the duct is a revolution of a curve $y=R(x)$ about $x$ axis, see Fig. 1. Since the area $a(x)$ is no longer constant, the pressure at the duct wall exerts a force normal to the wall onto the fluid. And this gives a contribution to the momentum in the central axis direction.

Let $R(x) \in C^{2}$ be the radius of the cross section, $a(x)=\pi R^{2}(x)$ be the area of the cross section. For any fixed $x_{0}$, we denote the disk bounded by the duct and the plane $x=x_{0}$ by $D_{x_{0}}$. Then for any point on the boundary of $D_{x}$, we can use $F_{x}$ to denote the $x$ component of the force exerted by the wall on the fluid in the $x$ direction. It's easy to see it does not depend on which point we choose. Similarly,

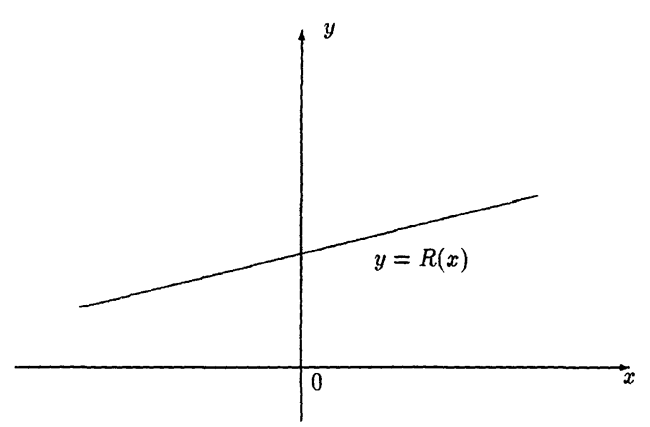

Fig. 1. 
the $x$ component of the unit normal vecto $\vec{n}_{x}$ is also well defined on the boundary of $D_{x}$. By simple calculation, we have

$$
\vec{n}=\left(R^{\prime}\left(1+\left(R^{\prime}\right)^{2}\right)^{-\frac{1}{2}},-\left(1+\left(R^{\prime}\right)^{2}\right)^{-\frac{1}{2}}\right),
$$

and so we get

$$
\begin{aligned}
F_{x} & =\vec{n}_{x} p \\
& =R^{\prime}\left(1+\left(R^{\prime}\right)^{2}\right)^{-\frac{1}{2}} p .
\end{aligned}
$$

Further, the total force acting on the boundary of the disk $D_{x}$ is given by

$$
\begin{aligned}
2 \pi R F_{x} & =2 \pi R R^{\prime}\left(1+\left(R^{\prime}\right)^{2}\right)^{-\frac{1}{2}} p \\
& =a^{\prime}\left(1+\left(R^{\prime}\right)^{2}\right)^{-\frac{1}{2}} p .
\end{aligned}
$$

Keeping these in mind, we can now derive the equations for the conservation of mass and momentum. For any $x_{1}<x_{2}$, consider the mass in the region bounded by $D_{x_{1}}$ and $D_{x_{2}}$. By conservation of mass, we have

$$
\begin{aligned}
\frac{d}{d t} \int_{x_{1}}^{x_{2}} \rho a d x & =-\left\{\left.(\rho u a)\right|_{x_{2}}-\left.(\rho u a)\right|_{x_{1}}\right\} \\
& =\int_{x_{1}}^{x_{2}}(\rho u a)_{x} d x,
\end{aligned}
$$

where $\left.(\cdot)\right|_{x_{0}}$ denotes the value of "." at $x=x_{0}$. Since $x_{1}$ and $x_{2}$ are arbitrary, we have

$$
(\rho a)_{t}+(\rho u a)_{x}=0
$$

in the weak sense.

By the conservation of momentum and (8), we have

$$
\begin{aligned}
\frac{d}{d t} \int_{x_{1}}^{x_{2}} \rho u a d x & =-\left\{\left.\left(\rho u^{2} a+p a\right)\right|_{x_{2}}-\left.\left(\rho u^{2} a+p a\right)\right|_{x_{1}}\right\}+\int_{x_{1}}^{x_{2}} p a^{\prime}\left(1+\left(R^{\prime}\right)^{2}\right)^{-\frac{1}{2}} d x \\
& =-\int_{x_{1}}^{x_{2}}\left\{\left(\rho u^{2} a+p a\right)_{x}-p a^{\prime}\left(1+\left(R^{\prime}\right)^{2}\right)^{-\frac{1}{2}}\right\} d x,
\end{aligned}
$$

or

$$
(\rho u a)_{t}+\left(\rho u^{2} a+p a\right)_{x}-p a^{\prime}\left(1+\left(R^{\prime}\right)^{2}\right)^{-\frac{1}{2}}=0,
$$

in the weak sense.

Now if we assume that $R^{\prime}(x)$ is sufficiently small, we have $\left(1+\left(R^{\prime}\right)^{2}\right)^{-\frac{1}{2}} \approx 1$, then Eqs. (9) and (10) are Eqs. (2) when $a(x)=x^{2}$, i.e., $R(x)^{\circ}=x$. Since when $R(x)=x$, the assumption of $R^{\prime}(x)$ being sufficiently small is violated. That is, Eqs. (2) are not good mathematical approximations for this physical model.

Now we consider spherically symmetric flow in $R^{3}$. As we will show later, Eqs. (2) are exact conservation laws of mass and momentum in this case. Here spherical symmetry means that the scalar quantities (pressure, density, etc.) are functions of radius $r$ and time $t$ only, while the velocity $\vec{u}$ points radially outwards or inwards.

In order to derive the equations easily, we consider the fluid in a region $D$ bounded by two co-centered hemispheres with radii $r_{1}$ and $r_{2}$ respectively and $x-y$ plane, where $r_{1}<r_{2}$. Without loss of generality, we assume this region is above the $x-y$ plane. Let $\rho$ be the density function of the gas; by the conservation of 
mass, we have

$$
\frac{d}{d t} \iiint_{D} \rho d V+\iint_{S_{2}} \rho u d A-\iint_{S_{1}} \rho u d A=0,
$$

where $d V$ and $d A$ are volume element and the area element respectively $S_{l}$ is the surface of the hemisphere with radius $r_{i}, i=1,2$. By the spherical symmetry, we have

$$
\int_{r_{1}}^{r_{2}}\left\{\left(\rho r^{2}\right)_{t}+\left(\rho u r^{2}\right)_{r}\right\} d r=0 .
$$

Since $r_{1}$ and $r_{2}$ are arbitrary, we have

$$
\left(r^{2} \rho\right)_{t}+\left(r^{2} \rho u\right)_{r}=0,
$$

in the weak sense. And this is the first equation of (2).

Now we consider the conservation of the momentum. By spherical symmetry, we can only need to consider the conservation of its $z$ component. For any radial vector $\vec{r}$, let $\theta$ be the angle between $\vec{r}$ and $z$ axis. For any point in the region $D$, the $z$ component of the momentum is $\rho u \cos \theta, 0 \leqq \theta \leqq \frac{\pi}{2}$. Thus we have

$$
\begin{aligned}
& \frac{d}{d t} \iiint_{D} \rho u \cos \theta d V+\iint_{S_{2}}(\rho u \cos \theta \cdot u+p \cos \theta) d S \\
& \quad-\iint_{S_{1}}(\rho u \cos \theta \cdot u+p \cos \theta) d S-\iint_{B} p d S=0,
\end{aligned}
$$

where $B$ is the base of $D$ on the $x-y$ plane. Since $\rho, u$ and $p$ are independent of $\theta$, and $\iint_{S_{l}} \cos \theta d S=\pi r_{i}^{2}, i=1,2$, we have

$$
\int_{r_{1}}^{r_{2}}\left\{\left(r^{2} \rho u\right)_{t}+\left(r^{2} \rho u^{2}+r^{2} p\right)_{r}\right\} d r=\int_{r_{1}}^{r_{2}} 2 r p d r .
$$

Since $r_{1}$ and $r_{2}$ are arbitrary, we have

$$
\left(r^{2} \rho u\right)_{t}+\left(r^{2} \rho u^{2}+r^{2} p\right)_{r}=2 r p,
$$

in the weak sense. This is the $2^{\text {nd }}$ equation of (2).

Thus Eqs. (2) are just the conservation laws of mass and momentum for the spherical symmetric flow in $R^{3}$.

\section{Solutions to Generalized Riemann Problem}

In this section, we will construct solutions to the generalized Riemann problem (2) and (6) when $|u|$ is sufficiently small and $p(\rho)=\rho$. The solutions for general cases were discussed in [7], and the following construction follows from [7] also.

Before solving generalized Riemann problem (2) and (6), we review some basic results about Riemann problems when $a(x)$ is constant, i.e., Eqs. (4) with initial data given by

$$
(u, \rho)= \begin{cases}\left(u_{l}, \rho_{l}\right), & x<0, \\ \left(u_{r}, \rho_{r}\right), & x>0 .\end{cases}
$$


By transforming $(u, \rho)$ to the Riemann invariants $(r(u, \rho), s(u, \rho))$, which are defined by $\mathscr{R}_{2} \cdot \nabla r=0$ and $\mathscr{R}_{1} \cdot \nabla s=0$, we have

$$
\begin{aligned}
& r_{t}+\lambda_{1} r_{x}=0, \\
& s_{t}+\lambda_{2} s_{x}=0,
\end{aligned}
$$

where $r=\frac{u-\ln \rho}{2}, s=\frac{u+\ln \rho}{2}$, and $\mathscr{R}_{i}, \lambda_{l}$ are defined in Sect. $1, i=1,2$.

By the definition of rarefaction wave, we can define the $1^{\text {st }}$ and $2^{\text {nd }}$ rarefaction wave curves as follows:

$$
\begin{aligned}
& R_{1}\left(u_{l}, \rho_{l}\right)=\left\{(u, \rho) \mid r \geqq r_{l}, s=s_{l}\right\}, \\
& R_{2}\left(u_{r}, \rho_{r}\right)=\left\{(u, \rho) \mid r=r_{r}, s \leqq s_{r}\right\},
\end{aligned}
$$

where $(r, s)=(r(u, \rho), s(u, \rho)),\left(r_{i}, s_{i}\right)=\left(r\left(u_{i}, \rho_{i}\right), s\left(u_{i}, \rho_{l}\right)\right)$ with $i=l, r$. Here $R_{1}\left(u_{l}, \rho_{l}\right)$ is the set of states $(u, \rho)$ which can be connected to the left state $\left(u_{l}, \rho_{l}\right)$ by rarefaction waves of the $1^{\text {st }}$ family, and $R_{2}\left(u_{r}, \rho_{r}\right)$ is the set of states $(u, \rho)$ which can be connected to the right state $\left(u_{r}, \rho_{r}\right)$ by rarefaction waves of the $2^{\text {nd }}$ family.

If $(u, \rho)$ is connected to $\left(u_{0}, \rho_{0}\right)$ by a single shock, by Rankine-Huguniot jump condition, we have

$$
\begin{gathered}
{[\rho u]=\sigma(u, \rho)[\rho],} \\
{\left[\rho u^{2}+\rho\right]=\sigma(u, \rho)[\rho u],}
\end{gathered}
$$

where $[\cdot]$ denote the difference between value "." on the right of the shock and the one of the left and $\sigma(u, \rho)$ is the speed of the shock. To satisfy the entropy condition, we have to ensure that the $i^{\text {th }}$ characteristics enter the shocks of the $i^{\text {th }}$ family from both sides, i.e., $\lambda_{l}\left(u_{r}, \rho_{r}\right)<\sigma(u, \rho)<\lambda_{i}\left(u_{l}, \rho_{l}\right)$ for the $i^{\text {th }}$ shock, $i=1,2$. Thus, we can define the $1^{\text {st }}$ and $2^{\text {nd }}$ shock curves as follows:

$$
\begin{aligned}
& S_{1}\left(u_{l}, \rho_{l}\right)=\left\{(u, \rho) \mid u_{l}-u=\left(\frac{\rho}{\rho_{l}}\right)^{\frac{1}{2}}-\left(\frac{\rho_{l}}{\rho}\right)^{\frac{1}{2}}, \rho_{l} \leqq \rho\right\}, \\
& S_{2}\left(u_{r}, \rho_{r}\right)=\left\{(u, \rho) \mid u-u_{r}=\left(\frac{\rho}{\rho_{r}}\right)^{\frac{1}{2}}-\left(\frac{\rho_{r}}{\rho}\right)^{\frac{1}{2}}, \rho_{r} \leqq \rho\right\},
\end{aligned}
$$

where $S_{i}(\cdot, \cdot)$ has similar meaning as $R_{l}(\cdot, \cdot), i=1,2$. To solve the Riemann problem (4) and (11), we define

$$
\begin{aligned}
& T_{1}\left(u_{l}, \rho_{l}\right)=R_{1}\left(u_{l}, \rho_{l}\right) \cup S_{1}\left(u_{l}, \rho_{l}\right), \\
& T_{2}\left(u_{r}, \rho_{r}\right)=R_{2}\left(u_{r}, \rho_{r}\right) \cup S_{2}\left(u_{r}, \rho_{r}\right) .
\end{aligned}
$$

It is well known that $T_{1}$ and $T_{2}$ are $C^{2}$ contact at points $\left(u_{l}, \rho_{l}\right)$ and $\left(u_{r}, \rho_{r}\right)$ respectively, and $T_{l}$ defines $\ln \rho$ as a monotonic function of $u$ for $i=1,2$. Furthermore, the $T_{2}$ curves are reflections of the $T_{1}$ curves with respect to axis $u=0$, and any $T_{1}$ curve always intersects any $T_{2}$ curve precisely once. Thus solution for the Riemann problem (4) and (11) is well defined and it consists of two hyperbolic waves (including waves with zero strengths). 
Now we come back to the generalized Riemann problem (2) and (6). In order to construct solutions to (2) and (6), we need to consider smooth stationary flow, i.e., $(u, \rho, a)$ satisfies

$$
\begin{aligned}
& (a \rho u)_{x}=0, \\
& \left(\frac{u^{2}}{2}+\ln \rho\right)_{x}=0,
\end{aligned}
$$

or

$$
\begin{aligned}
& \ln a+\ln \rho+\ln |u|=\text { constant } \\
& \frac{u^{2}}{2}+\ln \rho=\text { constant. }
\end{aligned}
$$

Keeping the area and state at $x_{-}=0^{-}$being $a_{l}$ and $\left(u_{l}, \rho_{l}\right)$; at $x_{+}=0^{+}$being $a_{r}$ and $\left(u_{r}, \rho_{r}\right)$, we vary the area smoothly and monotonically between $a_{l}$ and $a_{r}$. And we construct a smooth stationary flow between $x_{-}$and $x_{+}$. A straightforward computation shows that smooth stationary flow cannot become sonic, i.e., $\left|u_{l}\right|<1$ if and only if $\left|u_{r}\right|<1$. Now if we define $J\left(u_{l}, \rho_{1}, a_{l} ; a\right)$ as the set of states $(u, \rho)$ that can be connected to $\left(u_{l}, \rho_{l}\right)$ by a smooth stationary flow with $a(x)=a$, we have

$$
\begin{aligned}
& (\ln \rho+\ln |u|+\ln a)-\left(\ln \rho_{l}+\ln \left|u_{l}\right|+\ln a_{l}\right)=0, \\
& \left(\ln \rho+\frac{u^{2}}{2}\right)-\left(\ln \rho_{l}+\frac{u_{l}^{2}}{2}\right)=0 .
\end{aligned}
$$

It is easy to show that $J\left(u_{l}, \rho_{l}, a_{l} ; a\right)$ is a curve which can be parametrized by $a$ in the range $\left(a^{0}, \infty\right)$, where

$$
a^{0}=\exp \left\{\ln a_{l}+\ln \left|u_{l}\right|+\frac{1-u_{l}^{2}}{2}\right\} .
$$

As $a$ tends to $a^{0},|u|$ tends to 1 . As $a$ tends to infinity, $|u|$ tends to zero if $\left|u_{l}\right|<1$ and it tends to infinity if $\left|u_{l}\right|>1$.

Since our discussion is valid irrespective of how close $x_{-}$and $x_{+}$are, in our generalized Riemann problem, the standing wave can be defined as $x_{+}-x_{-}$tends to zero of the solution corresponding to a smooth monotonic interpolation of $a$. Based on the above discussion, we define the $1^{\text {st }}$ wave curve $C_{1}\left(u_{l} \rho_{l}\right)$ as a curve in the phase plane $(u-\ln \rho$ plane), which consists of a continuous succession of components with increasing speeds. The components would be the $1^{\text {st }}$ shock curve $S_{1}, 1^{\text {st }}$ rarefaction wave curve $R_{1}$ and standing wave curve $J$. Any state on $C_{1}\left(u_{l}, \rho_{l}\right)$ can be connected ot $\left(u_{l}, \rho_{l}\right)$ by hyperbolic waves of the $1^{\text {st }}$ family or standing waves or both. The $2^{\text {nd }}$ wave curve $C_{2}\left(u_{r}, \rho_{r}\right)$ can be defined analogously.

To solve the generalized Riemann problem is equivalent to finding the intersection of $C_{1}\left(u_{l}, \rho_{l}\right)$ and $C_{2}\left(u_{r}, \rho_{r}\right)$, i.e., to find the set $\left\{\left(u_{m}, \rho_{m}\right)\right\}$ such that

$$
\left\{\left(u_{m}, \rho_{m}\right)\right\}=C_{1}\left(u_{l}, \rho_{l}\right) \cap C_{2}\left(u_{r}, \rho_{r}\right) .
$$

In order to locate the standing wave, the following rule must be satisfied: if $u_{m}>0$ the standing wave curve is on the $1^{\text {st }}$ wave curve, and if $u_{m}<0$ it is on the $2^{\text {nd }}$ wave curve, and it vanished for $u_{m}=0$. 
Throughtout this paper, we assume $|u|$ is sufficiently small and $a_{l}<a_{r}$. The we only need to find $C_{1}\left(u_{l}, \rho_{l}\right)$ when $u_{l}<1, a_{l}<a_{r}$, and $C_{2}\left(u_{r}, \rho_{r}\right)$ when $u_{r}>$ $-1, a_{l}<a_{r}$. It is easy to see such $C_{2}\left(u_{r}, \rho_{r}\right)$ curves are reflections of $C_{1}\left(u_{l}, \rho_{l}\right)$ curves when $u_{l}<1$ and $a_{l}>a_{r}$ with respect to axis $u=0$. So we only need to find $C_{1}\left(u_{l}, \rho_{l}\right)$ for two cases.

Firstly, we consider the case when $u_{l}<1$ and $a_{l}>a_{r}$. It was proved in [7] that $C_{1}\left(u_{l}, \rho_{l}\right)$ consists of three parts, i.e., $Q_{1}, Q_{2}$ and $Q_{3}$, where

$$
\begin{aligned}
& Q_{1}\left(u_{l}, \rho_{l}\right)=\left\{(u, \rho) \mid(u, \rho) \in T_{1}\left(u_{l}, \rho_{l}\right) \text { with } u<0\right\} \\
& Q_{2}\left(u_{l}, \rho_{l}\right)=\left\{(u, \rho) \mid(u, \rho)=J\left(u_{-}, \rho_{-}, a_{l} ; a_{r}\right) \text { with } 0<u_{-} \leqq \bar{u}\right\} \\
& Q_{3}\left(u_{l}, \rho_{l}\right)=\left\{(u, \rho) \mid(u, \rho) \in T_{1}\left(1, \rho_{1}\right) \text { with } u>1\right\}
\end{aligned}
$$

here $\left(u_{-}, \rho_{-}\right),(\bar{u}, \bar{\rho}) \in T_{1}\left(u_{l}, \rho_{l}\right)$ and $\left(1, \rho_{1}\right)=J\left(\bar{u}, \bar{\rho}, a_{l} ; a_{r}\right)$. Figure 2 shows an example for this case. It is straightforward to prove that $C_{1}\left(u_{l}, \rho_{l}\right)$ defines $\ln \rho$ as a monotonically decreasing function of $u$.

Similarly, for the case when $u_{l}<1, a_{l}<a_{r}$, the curve $C_{l}\left(u_{l}, \rho_{l}\right)$ consists of four parts, i.e., $Q_{1}, Q_{2}, Q_{3}$ and $Q_{4}$, where

$$
\begin{aligned}
& Q_{1}\left(u_{l}, \rho_{l}\right)=\left\{(u, \rho) \mid(u, \rho) \in T_{1}\left(u_{l}, \rho_{l}\right) \text { with } u \leqq 0\right\}, \\
& Q_{2}\left(u_{l}, \rho_{l}\right)=\left\{(u, \rho) \mid(u, \rho)=J\left(u_{-}, \rho_{-}, a_{l} ; a_{r}\right) \text { with } 0<u_{-} \leqq 1, u<1\right\}, \\
& Q_{3}\left(u_{l}, \rho_{l}\right)=\left\{(u, \rho) \mid(u, \rho)=J\left(u_{+}, \rho_{+}, a ; a_{r}\right) \text { with } a_{l}<a<a_{r}\right\}, \\
& Q_{4}\left(u_{l}, \rho_{l}\right)=\left\{(u, \rho) \mid(u, \rho) \in T_{1}\left(\overline{u^{r}}, \overline{\rho^{r}}\right) \text { with } u>u_{+}^{r}\right\},
\end{aligned}
$$

where $\left(u_{-}, \rho_{-}\right) \in T_{1}\left(u_{l}, \rho_{l}\right)\left(1, \rho_{1}\right) \in T_{1}\left(u_{l}, \rho_{l}\right),(\bar{u}, \bar{\rho})=J\left(1, \rho_{1}, a_{l} ; a\right)$ with $a_{l}<$ $a<a_{r},\left(u_{+}, \rho_{+}\right)=S_{1}^{0}(\bar{u}, \bar{\rho}) \quad$ with $\quad \bar{u}>1,\left(\bar{u}^{r}, \bar{\rho}^{r}\right)=J\left(1, \rho_{1}, a_{l} ; a_{r}\right),\left(u_{+}^{r}, \rho_{+}^{r}\right)=$ $S_{1}^{0}\left(\left(\overline{u^{r}}, \overline{\rho^{r}}\right)\right.$ and $S_{1}^{0}\left(u_{0}, \rho_{0}\right)$ denotes the state $(u, \rho)$ which can be connected to the left state $\left(u_{0}, \rho_{0}\right)$ by a shock of the $1^{\text {st }}$ family with zero speed. Figure 3 shows an example for this case.

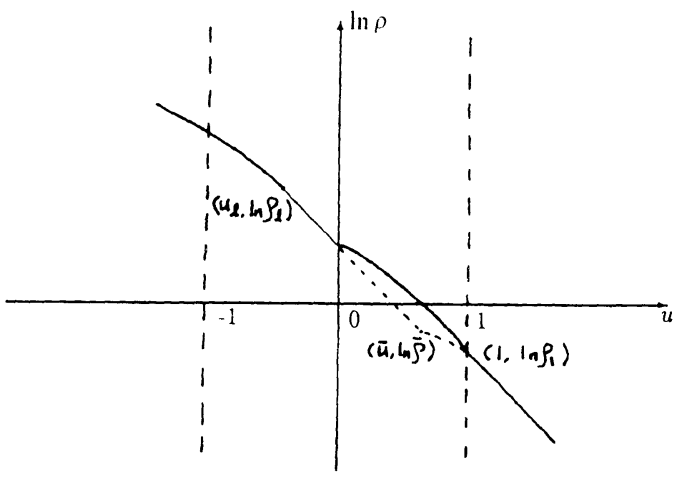

Fig. 2. 
It can be proved that $C_{1}\left(u_{l}, \rho_{l}\right)$ defines $\ln \rho$ as a monotonically decreasing function of $u$. For $\left|u_{l}\right|$ and $\left|u_{r}\right|$ being small, it can be shown that any $C_{1}\left(u_{l}, \rho_{1}\right)$ when $u_{l}<1, a_{l}<a_{r}$ and any $C_{2}\left(u_{r}, \rho_{r}\right)$ when $u_{r}>-1, a_{l}<a_{r}$ always intersect precisely once. Thus the Riemann problem can be solved by two hyperbolic waves and one standing wave. An example of this construction is shown in Figs. 4 and 5 .

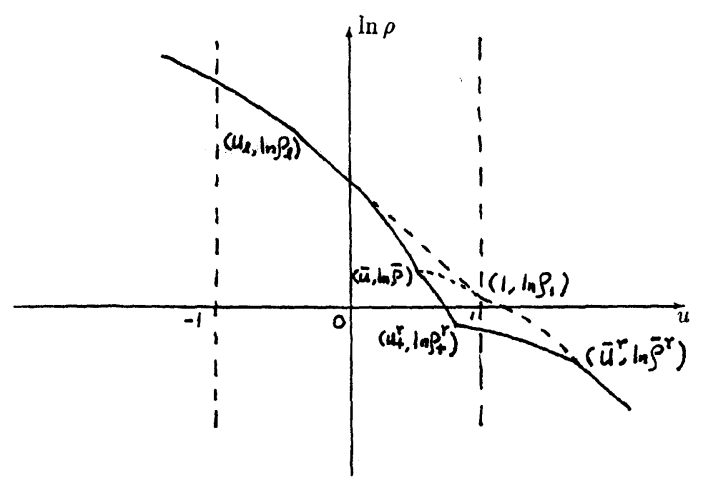

Fig. 3.

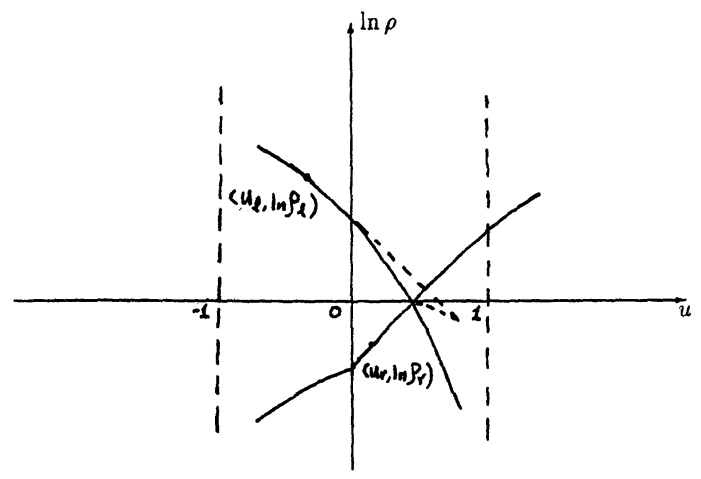

Fig. 4.

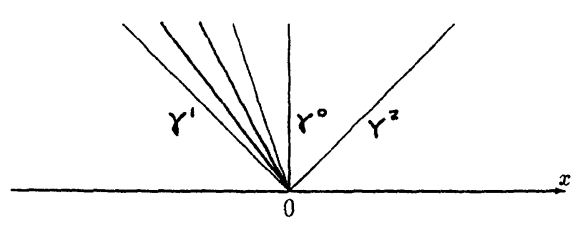

Fig. 5. 
Now we can restate a theorem from [7].

Given two states $\left(u_{l}, \rho_{l}, a_{l}\right)$ and $\left(u_{r}, \rho_{r}, a_{r}\right)$, if $a_{l}<a_{r}$ and $\left(u_{l}, \rho_{i}\right)$ is in a $\delta$ neighborhood of constant state $\left(0, \rho_{0}\right)$ for a small constant $\delta, i=l, r$. Then the Riemann problem of (2) and (6) can be solved by two hyperbolic waves, and one standing wave uniquely.

Theorem 2. Given two states $\left(u_{l}, \rho_{l}, a_{l}\right)$ and $\left(u_{r}, \rho_{r}, a_{r}\right)$, if $a_{l}<a_{r}$ and $\left(u_{i}, \rho_{i}\right)$ is in a $\delta$ neighborhood of constant state $\left(0, \rho_{0}\right)$ for a small constant $\delta, i=l, r$. Then the Riemann problem of (2) and (6) can be solved by two hyperbolic waves and one standing wave uniquely.

\section{Reduction to the Ideal Problem}

In this section, we will prove that the problem (2) and (3) can be reduced to the ideal problem diefined in Sect. 2 within the leading term of $\delta$. As shown in Sect. 4, the Riemann problem (2) and (6) can be solved uniquely by three waves, i.e., two hyperbolic waves and one standing wave. Thus we only need to prove that the wave interaction satisfies those conditions for the ideal problem. By the definition of the standing wave, we can estimate its Glimms's strength. If we assume two states of the Riemann problem (2) and (6) are connected by only one standing wave and

$$
a_{l}=x_{0}^{2}, \quad a_{r}=\left(x_{0}+\Delta x\right)^{2},
$$

$\delta$ and $\Delta x$ are sufficiently small, within the leading term with respect to $\delta$ and $\Delta x$, we have

$$
\begin{gathered}
\rho_{l}=\rho_{r}, \\
\left|u_{r}-u_{l}\right|=\frac{2 \Delta x}{x_{0}} u_{r} .
\end{gathered}
$$

As shown in Sect. 4, the hyperbolic waves are defined in the usual sense. Within the leading term with respect to $\delta$, we have $1^{\text {st }}$ wave curve given by

$$
s=s_{l}
$$

and the $2^{\text {nd }}$ wave curve given by

$$
r=r_{l}
$$

where $r$ and $s$ are the Riemann invariants defined in Sect. 4 and the subscript " $l$ " denotes the left state of the wave. By the entropy condition, when $u>u_{l}$ we have rarefaction waves while when $u<u_{l}$ we have shocks.

Now we can estimate the results when a hyperbolic wave interacts with a standing wave.

We consider a standing wave $\gamma^{0}$ sitting at $x=x_{0}$ with

$$
a(x)= \begin{cases}x_{0}^{2}, & x<x_{0} \\ \left(x_{0}+\Delta x\right)^{2}, & x>x_{0} .\end{cases}
$$

The following theorem was proved in [12]. 


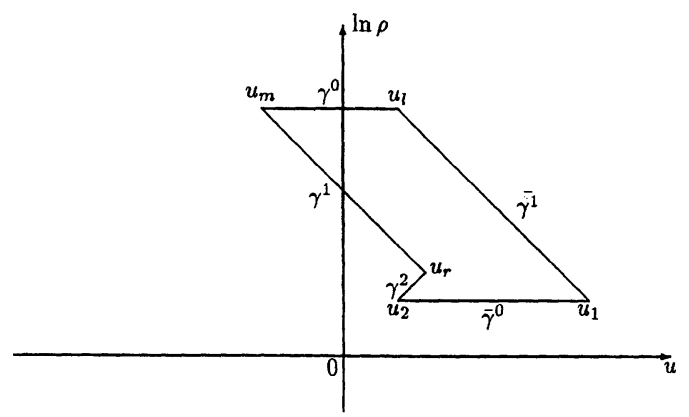

Fig. 6.

Theorem 3. Within the leading term with respect of $\delta$ and $\Delta x$, if there is a hyperbolic wave of the $1^{\text {st }}$ family $\gamma^{1}$ on the right of $\gamma^{0}$, after interaction we have

$$
\begin{gathered}
\gamma^{0}+\gamma^{1} \rightarrow \bar{\gamma}^{1}+\bar{\gamma}^{0}+\bar{\gamma}^{2}, \\
\left\|\bar{\gamma}^{1}\right\|=\left\|\gamma^{1}\right\|, \quad\left\|\bar{\gamma}^{2}\right\|=\frac{\Delta x}{x_{0}}\left\|\gamma^{1}\right\| .
\end{gathered}
$$

Similarly, if there is a hyperbolic wave of the $2^{\text {nd }}$ family $\gamma^{2}$ on the left of $\gamma^{0}$, after the interaction, we have

$$
\begin{gathered}
\gamma^{2}+\gamma^{0} \rightarrow \bar{\gamma}^{1}+\bar{\gamma}^{0}+\bar{\gamma}^{2}, \\
\left\|\bar{\gamma}^{2}\right\|=\left\|\gamma^{2}\right\|, \quad\left\|\bar{\gamma}^{1}\right\|=\frac{\Delta x}{x_{0}}\left\|\gamma^{2}\right\| .
\end{gathered}
$$

Proof. The proof is based on a case by case study; here we give a proof of one case for illustration.

Consider a rarefaction wave of the $1^{\text {st }}$ family $\gamma^{1}$ interacting a standing wave $\gamma^{0}$, we have

$$
\gamma^{0}+\gamma^{1} \rightarrow \bar{\gamma}^{1}+\bar{\gamma}^{0}+\bar{\gamma}^{2}
$$

Since all the estimates are within the leading term, as shown by Fig. 6, we have

$$
\begin{aligned}
& \left|\gamma^{0}\right|=\left|u_{r}-u_{m}\right|=\frac{2 \Delta x}{x_{0}+\Delta x} u_{l}, \\
& \left|\bar{\gamma}^{0}\right|=\left|u_{2}-u_{1}\right|=\frac{2 \Delta x}{x_{0}+\Delta x} u_{1} .
\end{aligned}
$$

Then

$$
\left|\gamma^{0}-\bar{\gamma}^{0}\right|=2\left|\bar{\gamma}^{2}\right|=2\left|\bar{\gamma}^{-1}-\gamma^{1}\right|
$$

i.e.,

$$
\begin{aligned}
\left|\bar{\gamma}^{-1}\right|-\left|\gamma^{1}\right|=\frac{\Delta x}{x_{0}+\Delta x}\left|u_{l}-u_{1}\right| & =\frac{\Delta x}{x_{0}+\Delta x}\left|\bar{\gamma}^{-1}\right|, \\
\left(x_{0}+\Delta x\right)\left|\gamma^{1}\right| & =x_{0}\left|\bar{\gamma}^{-1}\right|, \\
\left\|\bar{\gamma}^{-1}\right\| & =\left\|\gamma^{1}\right\| .
\end{aligned}
$$


Since

$$
\left|\bar{\gamma}^{2}\right|=\left|\bar{\gamma}^{-1}-\gamma^{1}\right|=\frac{\Delta x}{x_{0}+\Delta x}\left|\bar{\gamma}^{-1}\right|
$$

then

$$
\left\|\bar{\gamma}^{2}\right\|=\frac{\Delta x}{x_{0}}\left\|\bar{\gamma}^{-1}\right\|=\frac{\Delta x}{x_{0}}\left\|\gamma^{1}\right\| . \quad \text { QED }
$$

Note 2. All the equalities are within the leading term by assuming $\delta$ and $\Delta x$ being small.

Note 3. It was pointed out in [12] that shocks (rarefaction waves) of the 2 nd family reflect rarefaction waves (shocks) of the $1^{\text {st }}$ family, while shocks (rarefaction waves) of the $1^{\text {st }}$ family reflect shocks (rarefaction waves) of the $2^{\text {nd }}$ family. Thus the cancellation will occur due to the reflected waves of a same wave. And this kind of cancellation might overcome the generation of the reflected waves growing logarithmically. And we will prove that this is true under the assumptions of Theorem 1.

Since we assume $u(1, t) \equiv 0$ for $t \geqq 0$, in [8] the unchanged strength of the rebounded wave was proved in Lagrangian coordinates. It's easy to see that this is true for Euler coordinates by the symmetry of the shock curves with respect to $u=0$ for Eqs. (4). And it is obviously true when we consider the leading term with respect to $\delta$. Thus, we have shown that the Cauchy problem (2) and (3) can be reduced to the ideal problem within the leading term of $\delta$.

\section{The Difference Scheme}

In order to get a uniform bound for the solutions of the ideal problem, we will repose the problem in Glimm's scheme by solving a class of generalized Riemann problems (7) and (6). In this section, we define Glimm's scheme which can be used to construct solutions for the ideal problem. By assuming that the hyperbolic waves move at constant speeds, we can modify Glimm's scheme such that the cancellations between reflections are easier to calculate.

We assume that the absolute values of the speeds of the hyperbolic waves lie between $\left[\xi_{1}, \xi_{2}\right]$. Let $h$ be a mesh length in $x$, and let $k=\frac{h}{c_{0}}$ be the corresponding mesh length in $t, c_{0}>\xi_{2}$. Let a be any equidistributed sample sequence, $\mathbf{a} \equiv\left\{a_{j}\right\}_{j=1}^{\infty}, 0<a_{j} \leqq 1$. For $t=0$, we define

$$
\begin{aligned}
\rho_{0}^{h}(0, x) & =\rho_{0}(m h), \\
u_{0}^{h}(0, x) & =u_{0}(m h), \quad 1+(m-1) h<x<1+(m+1) h, \\
u_{0}^{h}(t, 1) & \equiv 0, \quad t \geqq 0, \\
a(x) & =(1+(m+1) h)^{2}, \quad 1+(m-1) h<x<1+(m+1) h,
\end{aligned}
$$

where $m \geqq 1$ is any odd integer. At mesh points $(1+(m+1) h, 0)$ and $(1,0)$, we can solve the Riemann problem by hyperbolic waves and the standing wave. 
Assume for induction that $\left(\rho^{h}(x, t), u^{h}(x, t)\right)$ has been defined for $t<i k$, when $i+m$ is odd, we define

$$
\begin{aligned}
\left(\rho^{h}, u^{h}\right)(x, i k) \equiv & \left(\rho^{h}, u^{h}\right)\left(2 m h+a_{l} h, i k-\right) \\
& 1+2 m h<x<1+(2 m+2) h \\
\left(\rho^{h}, u^{h}\right)(x, i k) \equiv & \left(\rho^{h}, u^{h}\right)\left(2 m h-a_{l} h, i k-\right) \\
& 1+(2 m+2) h<x<1+(2 m+4) h
\end{aligned}
$$

and

$$
u^{h}(1, t) \equiv 0, i k \leqq t<(i+1) k
$$

where $m$ is any integer such that $i+m$ is odd and $x \geqq 1$. lemmas.

By the above construction of the approximate solution, we have the following

Lemma 1. If all the hyperbolic waves are sitting at $(1+2 m h,(i-1) k)$ for $m \geqq 0$ when $i+m$ is odd, then we have

- if $a_{l} \in\left(0,1-v_{1}\right)$, then every nonvanishing hyperbolic wave will cross the corresponding standing wave and reflect another hyperbolic wave.

- if $a_{i} \in\left(1-v_{2}, 1\right]$, then the strengths and positions of all the waves in $[1, \infty) \times$ $[i k,(i+1) k)$ are the same as those in $[1, \infty) \times[(i-1) k, i k)$,

where $v_{1}=\frac{\xi_{1}}{c_{0}}$ and $v_{2}=\frac{\xi_{2}}{c_{0}}$. A similar statement holds when $i+m$ is even.

Lemma 2. If we denote the hyperbolic wave of the $1^{\text {st }}\left(2^{\text {nd }}\right)$ family sitting at $(1+m \Delta x, 0)$ by $\gamma_{m, 0}^{1}\left(\gamma_{m, 0}^{2}\right)$, where $\Delta x=2 h$, then under the conditions (A) and (B) we have, for any fixed $n>0$, when $\Delta x \ll 1$,

$$
\sum_{m=0}^{\infty} \frac{\Delta x}{1+m \Delta x}\left\{\sum_{l=1}^{n}\left\|\gamma_{m+l, 0}^{1}\right\|\right\} \leqq \bar{\delta}, \quad \sum_{m=0}^{\infty} \frac{\Delta x}{1+m \Delta x}\left\{\sum_{l=1}^{n}\left\|\gamma_{m+l, 0}^{2}\right\|\right\} \leqq \bar{\delta}
$$

and

$$
\sum_{m=0}^{\infty}\left\|\gamma_{m, 0}^{1}\right\| \ln (1+m \Delta x) \leqq \bar{\delta}, \quad \sum_{m=0}^{\infty}\left\|\gamma_{m, 0}^{2}\right\| \ln (1+m \Delta x) \leqq \bar{\delta}
$$

where $\bar{\delta}=O(\delta)$.

The Glimm's scheme given above can be used to prove the case correpsonding to the one mentioned in Remark 2. And we suggest that it may be useful when we consider the full nonlinear problem. But for the ideal problem correpsonding to our model problem in Theorem 1, we can use a simplifed scheme: If we denote the mesh length in the $x$ direction by $\Delta x$ and the one in the $t$ direction by $\Delta t$, then for any hyperbolic wave sitting at $(1+m \Delta x, i \Delta t)$, it will move to $(1+(m \pm 1) \Delta x$, $(i+1) \Delta t)$ at the next time step if it is of the $2^{\text {nd }}\left(1^{\text {st }}\right)$ family. That is, all hyperbolic waves of the $1^{\text {st }}$ family move at speed $-\Delta x / \Delta t$, and those of the $2^{\text {nd }}$ family move at speed $\Delta x / \Delta t$. We will use this simplified scheme in the following discussion. 


\section{Analysis of Cancellation in Reflections}

In this section we consider an ideal problem defined in Sect. 2 corresponding to the model problem in Theorem 1, and we will derive a formula showing how the hyperbolic waves on $T=N \Delta t$ depends on those $t=0$. In order to define reflections clearly, we introduce the concept of path in the solutions of our model problem. From now on, we use $(m, i)$ to denote the mesh point $(1+m \Delta x, i \Delta t)$ in the simplified scheme. We assume $N$ can be arbitrarily large but fixed and we consider the region $0 \leqq t \leqq N \Delta t, x \geqq 1$. Now we give the following definitions.

Definition 3. We define two kinds of elementary timelike paths, denoted by $I_{+}$ and $l_{-}$, where $l_{ \pm}$are straight lines connecting $(m, i)$ and $(m \pm 1, i+1)$. And we denote the end points and starting points of $l_{ \pm}$by

$$
\left(l_{ \pm}\right)^{0}=(m, i), \quad\left(l_{ \pm}\right)_{0}=(m \pm 1, i+1) .
$$

Definition 4. An ordered sequence of elementary paths $\imath=\left[l_{q}\right]_{q=1}^{p}$ is called a timelike path if

$$
\left(l_{q}\right)_{0}=\left(l_{q+1}\right)^{0}, \quad q=1,2, \ldots, p-1 .
$$

The product of $\left[l_{q}\right]_{q=1}^{p}$ denoted by $\mathrm{l}$ or $l_{1} \cdot l_{2} \cdots l_{p}$ is a polygonal line by connecting $\imath_{q} s$ at their common points. We define

$$
(l)^{0}=\left(l_{1}\right)^{0}, \quad(l)_{0}=\left(l_{p}\right)_{0} .
$$

Obviously, the product of an ordered sequence of timelike paths can be defined similarly.

Definition 5. For any timelike path $\mathrm{l}$, we define the degree of $\mathrm{l}$ by

$$
d(l)=d,
$$

if

$$
l=l_{1} \cdot l_{2} \cdots l_{d+1},
$$

where $l_{q}$ is a timelike path consisting of the same kind of elementary timelike paths, but $\imath_{q}$ and $\imath_{q+1}$ consist of different kinds. Also we define $\left(l_{q}\right)_{0}$ as the $q^{\text {th }}$ turning point of $l$, denoted by $T_{q}(l), q=1,2, \ldots, d$, and

$$
T(l)=\bigcup_{q=1}^{d} T_{q}(l) .
$$

Now we can define the reflections of any hyperbolic wave. And we will classify all the reflections by their orders according to the degrees of the paths to which they correspond.

Definition 6. For any hyperbolic wave $\gamma$ emitting from the mesh point $(m, i)$, and any timelike path $\mathrm{l}$ with

$$
(l)^{0}=(m, i), \quad(l)_{0}=(\bar{m}, \bar{i}),
$$


if

$$
d(l)=d \quad \text { and } \quad T(l)=\bigcup_{q=1}^{d}\left(m_{q}, i_{q}\right)
$$

then we define a wave $R(\gamma, l)$ at $(\bar{m}, \bar{i})$ with norm being $\left\{\prod_{q=1}^{d} \frac{\Delta x}{1+m_{q} \Delta x}\right\}\|\gamma\|$ as a $d^{\text {th }}$ reflection of $\gamma$ corresponding to the path $l$. If $l_{d+1}=\left[l_{+}\right]\left(\left[l_{-}\right]\right)$, then $R(\gamma, l)$ is of the $2^{\text {nd }}\left(1^{\text {st }}\right.$ family. Further, if $\gamma$ is a rarefaction wave (shock) of the $1^{\text {st }}$ family, then $R(\gamma, l)$ is a rarefaction wave (shock) when $d$ is odd, and it is a shock (rarefaction wave) when $d$ is even. Similarly, if $\gamma$ is a rarefaction wave (shock) of the $2^{\text {nd }}$ family, then $R(\gamma, \imath)$ is a shock (rarefaction wave) when $d$ is odd, and it is a rarefaction wave (shock) when $d$ is even. " $d$ " is also called as the order of the reflection $R(\gamma, l)$.

To classify reflections of a hyperbolic wave, we need the following definitions. Definition 7. For any hyperbolic wave $\gamma$ emitting from the mesh point $(m, i)$, we define

$$
R_{\gamma}^{q}=\bigcup_{\{l d d(l)=q\}} R(\gamma, l), \quad\left(R_{\gamma}^{q}\right)_{\bar{m}}^{\bar{i}}=\bigcup_{\{\bar{l}: d(\bar{\imath})=q\}} R(\gamma, \bar{l})
$$

and

$$
\left\|\left(R_{\gamma}^{q}\right)_{\bar{m}}^{i}\right\|=\sum_{\{i: d(\bar{l})=q\}}\|R(\gamma, \bar{l})\|
$$

where the unions and the sum are taken over all timelike paths $l$ and $\bar{l}$, with $(l)^{0},(\bar{l})^{0}=(m, i)$ and $(\bar{i})_{0}=(\bar{m}, \bar{i})$ in the region bounded by $x \geqq 1$ and $0 \leqq t$ $\leqq N k$.

Definition 8. For any hyperbolic wave $\gamma$ emitting from the mesh point $(m, i)$ and any mesh point $(\bar{m}, \bar{i})$, we define

$$
\gamma_{\bar{m}, \bar{i}}^{q, q+2}=\max \left\{\left\|\left(R_{\gamma}^{q}\right)_{\bar{m}}^{\bar{i}}\right\|-\left\|\left(R_{\gamma}^{q+2}\right)_{\bar{m}}^{\bar{i}}\right\|, 0\right\}
$$

Then at the mesh point $(\bar{m}, \bar{i})$, we define $\left(R_{\gamma}^{q} \backslash R_{\gamma}^{q+2}\right)_{\bar{m}}^{\bar{i}}$ as a hyperbolic wave which is of the same family and same kind as $\left(R_{\gamma}^{q}\right)_{\bar{m}}^{\bar{i}}$, and

$$
\left\|\left(R_{\gamma}^{q} \backslash R_{\gamma}^{q+2}\right)_{\bar{m}}^{\bar{i}}\right\|=\gamma_{\bar{m}, \bar{l}}^{q, q+2}
$$

And we define

$$
R_{\gamma}^{q} \backslash R_{\gamma}^{q+2}=\bigcup_{(\bar{m}, \bar{l})}\left(R_{\gamma}^{q} \backslash R_{\gamma}^{q+2}\right)_{\bar{m}}^{\bar{i}}
$$

for all possible $(\bar{m}, \bar{i})$. 
Definition 9. For any hyperbolic wave $\gamma$ emitting from $(m, i)$, we define

$$
\begin{aligned}
& R T^{0}(\gamma)=\sum_{q, j>0}\left\|\left(R_{\gamma}^{q} \backslash R_{\gamma}^{q+2}\right)_{0}^{j}\right\|, \\
& R T^{1}(\gamma)=\sum_{q, n>0}\left\|\left(R_{\gamma}^{q} \backslash R_{\gamma}^{q+2}\right)_{n}^{N}\right\|,
\end{aligned}
$$

where the sum is over all $\left(R_{\gamma}^{q} \backslash R_{\gamma}^{q+2}\right)_{n}^{N}$ are of the $1^{\text {st }}$ family, and

$$
R T^{2}(\gamma)=\sum_{q, n}\left\|\left(R_{\gamma}^{q} \backslash R_{\gamma}^{q+2}\right)_{n}^{N}\right\|
$$

where the sum is over all $\left(R_{\gamma}^{q} \backslash T_{i}^{q+2}\right)_{n}^{N}$ is of the $2^{\text {nd }}$ family, and the sums are taken over all possible $q, j$ and $n$.

Now we assume that there is only one hyperbolic wave $\gamma$ in the simplified scheme for the ideal model problem initially. Since we consider the behaviour of $\gamma$ and its reflections for any time, the orders of reflections due to $\gamma$ can be arbitrarily large. As pointed out in Remark 1, the sum of the total reflection cannot be bounded unless cancellations between reflected waves are taken into account. In order to calculate this kind of cancellation, we will introduce reflection potential. In fact, the estimation of the reflection potential is crucial to prove the following main lemma.

Lemma 3. We consider a hyperbolic wave $\gamma$ emitting from mesh point $(m, 0)$ in the simplified scheme. For any given $N>0$, if $\Delta x \ll 1$ and $\gamma$ is a hyperbolic wave of the $2^{\text {nd }}$ family, then

$$
R T^{l}(\gamma) \leqq \bar{G}\|\gamma\|, \quad i=0,1,2
$$

If $\gamma$ is a hyperbolic wave of the $1^{\text {st }}$ family, then

$$
R T^{l}(\gamma) \leqq \bar{G}\{2+\ln (1+m \Delta x)\}\|\gamma\|, \quad i=0,1,2,
$$

where $\bar{G}>0$ is a constant independent of $N, m$ and $\Delta x$.

Before defining the reflection potential, we give two more remarks on the cancellation.

Remark 5. For any hyperbolic wave emitting from $t=0$, we consider the reflected shocks and rarefaction waves separately. Since they will cancel each other if they reach a same mesh point, we can estimate the remainder of this kind of cancellation. That is, we estimate how much strength of shocks and rarefaction waves can reach $t=N k, x \geqq 1$ and $0 \leqq t \leqq N k, x=1$ after cancellation.

Remark 6. We notice that the $i^{\text {th }}$ reflected shocks (rarefaction waves) will make cancellation with the $(i+2)^{\text {nd }}$ reflected rarefaction waves (shocks) of a same original wave, if they meet at a same mesh point. We will prove the upper bounds for the remainders of this kind of cancellation due to a $2^{\text {nd }}$ hyperbolic wave form a geometrical series. Thus the sum can be bounded uniformly.

We consider a hyperbolic wave $\gamma$ of the $2^{\text {nd }}$ family emitting from the mesh point $(m, 0)$ initially, where $m \geqq 0$ is any integer. In order to estimate the reflection 
of higher order, we define reflection potential at any mesh point $(m, i)$ on which $\left\|\left(R_{\gamma}^{1} \backslash R_{\gamma}^{3}\right)_{m}^{i}\right\| \neq 0$. It represents how strongly $R_{\gamma}^{1} \backslash R_{\gamma}^{3}$ will reflect again.

Definition 10. If the hyperbolic wave $\gamma$ is of the $2^{\text {nd }}$ family, we define the reflection potential of $\gamma$ by $P(\gamma)$, where

$$
P(\gamma)=\sum_{l, m \geqq 2} \frac{\Delta x}{1+m \Delta x}\left\|\left(R_{\gamma}^{1} \backslash R_{\gamma}^{3}\right)_{m}^{i}\right\|+\sum_{i}\left\|\left(R_{\gamma}^{1} \backslash R_{\gamma}^{3}\right)_{1}^{l}\right\|,
$$

here $m \geqq 0$ and $0 \leqq i \leqq N$.

We are going to prove $P(\gamma) \leqq \eta\|\gamma\|$ for some constant $\eta<1$, where $\eta$ is independent of $N$ and $\Delta x$. Now we prove some lemmas first.

Lemma 4. If $\gamma$ emits from the mesh point $(0,0)$, when $\Delta x \ll 1$, we have

$$
p \Delta x \leqq 0.9
$$

where $p=\sup \left\{i:\left\|\left(R_{\gamma}^{1} \backslash R_{\gamma}^{3}\right)_{1}^{i}\right\| \neq 0\right\}$.

Proof. Let $\alpha_{p} \in R\left(\gamma, l_{0}\right)$ be the hyperbolic wave of the $1^{\text {st }}$ family with $d\left(l_{0}\right)=1$, $T_{1}\left(l_{0}\right)=(p, p)$ and $\left(l_{0}\right)_{0}=(1,2 p-1)$. We divide $\left(R_{\gamma}^{3}\right)_{1}^{2 p-1}$ into two families:

- The first family consists of those $R(\gamma, l)$ with $T_{2}(l)=(0, \cdot)$. We denote the total norm of this family by $I_{p, 0}^{1}\|\gamma\|$.

- The second family consists of those $R(\gamma, l)$ with $T_{2}(l)=(j, \cdot), j \geqq 1$. The total norm of this family is denoted by $I_{p, 0}^{2}\|\gamma\|$.

We have

$$
\begin{gathered}
I_{p, 0}^{1}=\sum_{i=1}^{p-1} \frac{(\Delta x)^{2}}{(1+j \Delta x)(1+(p-j) \Delta x)} \\
=\frac{2 \Delta x}{2+p \Delta x}\left\{\sum_{i=1}^{p-1} \frac{\Delta x}{1+i \Delta x}\right\} \\
=\frac{2 \Delta x}{2+p \Delta x} \ln (1+p \Delta x)+O(\Delta x)^{2}, \\
I_{p, 0}^{2}=\sum_{j=2}^{p-1} \frac{\Delta x}{1+j \Delta x} \sum_{i=1}^{j-1} \frac{(\Delta x)^{2}}{(1+i \Delta x)(1+(p+i-j) \Delta x)} \\
>\sum_{i=2}^{p-1} \frac{(\Delta x)^{2}}{(1+(p-i) \Delta x)(1+i \Delta x)}-\frac{\Delta x}{1+(p-1) \Delta x} \sum_{i=2}^{p-1} \frac{\Delta x}{1+i \Delta x} \\
>\frac{2 \Delta x}{2+p \Delta x} \ln (1+p \Delta x)-\frac{\Delta x}{1+p \Delta x} \ln (1+p \Delta x)+O(\Delta x)^{2} .
\end{gathered}
$$


Combining (12) and (13), we have

$$
\begin{aligned}
\left\|\alpha_{p}\right\| /\|\gamma\|-I_{p, 0}^{1}-I_{p, 0}^{2} & <\frac{\Delta x}{1+p \Delta x}-\frac{4 \Delta x}{2+p \Delta x} \ln (1+p \Delta x) \\
& +\frac{\Delta x}{1+p \Delta x} \ln (1+p \Delta x)+O(\Delta x)^{2}
\end{aligned}
$$

We can check that when $p \Delta x \geqq 0.9, \Delta x \ll 1$, the right-hand side of (14) is less than $-\varepsilon_{1} \Delta x$, where $\varepsilon_{1}>0$ is a constant independent of $N$ and $\Delta x$. Thus, we can choose $p \Delta x=0.9$. QED

Lemma 5. When $\Delta x \ll 1,\left\|\left(R_{\gamma}^{1} \backslash R_{\gamma}^{3}\right)_{m}^{i}\right\| \equiv 0$ for $(m, i) \in \Omega^{c}$, where

$$
\begin{aligned}
& \Omega \equiv\left\{(x, t): 0 \leqq t \leqq 2 p \Delta t, 1 \leqq x \leqq 1+\left[\frac{t}{\Delta t}\right] \Delta x\right. \\
&\text { or } \left.t>2 p \Delta t, 1+\left(\left[\frac{t}{\Delta t}\right]-2 p\right) \Delta x \leqq x \leqq 1+\left[\frac{t}{\Delta t}\right] \Delta x\right\},
\end{aligned}
$$

$p$ is defined in Lemma $4,\left[\right.$ • ] denotes the largest integer less than " . " and $\Omega^{c}$ is the complement of $\Omega$.

Proof. For $n \geqq p$, we calculate the cancellation between $\alpha_{n} \in R\left(\gamma, l_{0}\right)$, with $d\left(l_{0}\right)=1, T_{1}\left(l_{0}\right)=(n, n)$ and $\left(l_{0}\right)_{0}=(n-p+1, n+p-1)$, and $\left(R_{\gamma}^{3}\right)_{n-p+1}^{n+p-1}$. As in the proof of Lemma 4, we also divide $\left(R_{\gamma}^{3}\right)_{n-p+1}^{n+p-1}$ into two families, with their norms denoted by $I_{n, 1}^{1}\|\gamma\|$ and $I_{n, 1}^{2}\|\gamma\|$. The meaning of $I_{n, 1}^{1}$ and $I_{n, 1}^{2}$ are similar as $I_{n, 0}^{1}$ and $I_{n, 0}^{2}$ in Lemma 4 respectively.

$$
\begin{aligned}
& I_{n, 1}^{1}=\sum_{i=1}^{p-1} \frac{(\Delta x)^{2}}{(1+i \Delta x)(1+(n-i) \Delta x)} \\
& =\frac{\Delta x}{2+n \Delta x} \sum_{i=1}^{p-1}\left\{\frac{\Delta x}{1+i \Delta x}+\frac{\Delta x}{1+(n-i) \Delta x}\right\} \\
& >\frac{\Delta x}{2+n \Delta x}\left\{\ln (1+p \Delta x)+\ln \left\{\frac{1+n \Delta x}{1+(n-p) \Delta x}\right\}\right\}+\frac{1}{1+n \Delta x} O(\Delta x)^{2}, \\
& I_{n, 1}^{2}=\sum_{j=2}^{p-1} \frac{\Delta x}{1+j \Delta x} \sum_{i=1}^{j-1} \frac{(\Delta x)^{2}}{(1+i \Delta x)(1+(n+i-j) \Delta x)} \\
& +\sum_{j=p}^{n-1} \frac{\Delta x}{1+j \Delta x} \sum_{i=1}^{p-1} \frac{(\Delta x)^{2}}{(1+i \Delta x)(1+(n+i-j) \Delta x)} \\
& =\sum_{j=n-p+2}^{n-1} \frac{(\Delta x)^{2}}{1+j \Delta x} \sum_{i=1}^{j-1} \frac{\Delta x}{(1+i \Delta x)(1+(n+i-j) \Delta x)} \\
& >\sum_{i=1}^{p-2} \frac{(\Delta x)^{2}}{(1+(n-i) \Delta x)(1+i \Delta x)}-\frac{\Delta x}{1+(n-1) \Delta x} \sum_{l=n-p+2}^{n-1} \frac{\Delta x}{1+i \Delta x}
\end{aligned}
$$




$$
\begin{aligned}
> & \frac{\Delta x}{2+n \Delta x}\left\{\ln (1+p \Delta x)+\ln \left\{\frac{1+n \Delta x}{1+(n-p) \Delta x}\right\}\right\} \\
& -\frac{\Delta x}{1+n \Delta x} \ln \left\{\frac{1+n \Delta x}{1+(n-p) \Delta x}\right\}+\frac{1}{1+n \Delta x} O(\Delta x)^{2} .
\end{aligned}
$$

Let

$$
\begin{aligned}
L_{n}(n \Delta x)= & \|\alpha\| /\|\gamma\|-I_{n, 1}^{1}-I_{n, 1}^{2} \\
< & \frac{\Delta x}{1+n \Delta x}-\frac{2 \Delta x}{2+n \Delta x}\left\{\ln (1+p \Delta x)+\ln \left\{\frac{1+n \Delta x}{1+(n-p) \Delta x}\right\}\right\} \\
& +\frac{\Delta x}{1+n \Delta x} \ln \left\{\frac{1+n \Delta x}{1+(n-p) \Delta x}\right\}+\frac{1}{1+n \Delta x} O(\Delta x)^{2} \\
= & \mathscr{L}_{n}(n \Delta x) \Delta x .
\end{aligned}
$$

To prove the lemma is equivalent to prove that $L_{n}(n \Delta x)<0$ for $n \geqq p$ and $\Delta x \ll 1$. By calculation, we can prove that $\mathscr{L}_{n}(n \Delta x)<\frac{-\varepsilon_{2} \Delta x}{1+n \Delta x}$ for $n \geqq p$, where $\varepsilon_{2}>0$ is a constant independent of $N$ and $\Delta x$. QED

Since we have proved that $\left\|\left(R_{\gamma}^{1} \backslash R_{\gamma}^{3}\right)_{m}^{i}\right\| \equiv 0$ for $(m, i) \in \Omega^{c}$. Thus $P(\gamma)$ is equal to the total norms of the set of hyperbolic waves reflected by the nonvanishing $R_{\gamma}^{1} \backslash R_{\gamma}^{3}$ in $\Omega$.

Firstly, we estimate $R_{\gamma^{\prime}}^{1} \backslash R_{\gamma^{\prime}}^{3}$ at each point in $\Omega$.

Lemma 6. For any mesh point $(m, 2 i-m) \in \Omega$, and $\Delta x \ll 1$, we have

$$
\begin{aligned}
& \left\|\left(R_{\gamma}^{1} \backslash R_{\gamma}^{3}\right)_{m}^{2 l-m}\right\| \leqq \frac{\Delta x}{(1+i \Delta x)(1+q \Delta x)}\|\gamma\|, \quad \text { as } \quad 0 \leqq q \Delta x \leqq 0.5, \\
& \left\|\left(R_{\gamma}^{1} \backslash R_{\gamma}^{3}\right)_{m}^{2 i-m}\right\| \leqq \frac{3 \Delta x}{4(1+i \Delta x)(1+q \Delta x)}\|\gamma\|, \quad \text { as } \quad 0.5 \leqq q \Delta x \leqq 0.9,
\end{aligned}
$$

where $q=i-m$.

Proof. For $0 \leqq q \Delta x \leqq 0.5$,

$$
\begin{aligned}
\left\|\left(R_{\gamma}^{1} \backslash R_{\gamma}^{3}\right)_{m}^{2 i-m}\right\| /\|\gamma\| & <\frac{\Delta x}{1+i \Delta x}-\sum_{j=1}^{q} \frac{(\Delta x)^{2}}{(1+j \Delta x)(1+(i-j+1) \Delta x)} \\
& <\frac{\Delta x}{1+i \Delta x}-\frac{q(\Delta x)^{2}}{(1+i \Delta x)(1+q \Delta x)} \\
& =\frac{\Delta x}{(1+q \Delta x)(1+i \Delta x)} .
\end{aligned}
$$


For $0.5 \leqq q \Delta x \leqq 0.9$, we have to consider the cancellation from all $R_{\gamma}^{3}$ not just from the rebounded waves. By (15) and (16), we have

$$
\begin{aligned}
\left\|\left(R_{y}^{1} \backslash R_{\gamma}^{3}\right)_{m}^{2 i-m}\right\| /\|\gamma\| & \\
< & \frac{\Delta x}{1+i \Delta x}-\frac{2 \Delta x}{2+i \Delta x}\left\{\operatorname{In}(1+q \Delta x)+\ln \left\{\frac{1+i \Delta x}{1+(i-q) \Delta x}\right\}\right\} \\
& +\frac{\Delta x}{1+i \Delta x} \ln \left\{\frac{1+i \Delta x}{1+(i-q) \Delta x}\right\}+\frac{1}{1+i \Delta x} O(\Delta x)^{2} \\
= & \frac{\Delta x}{1+i \Delta x}-\frac{2 \Delta x}{2+i \Delta x} \ln (1+q \Delta x)-\frac{i(\Delta x)^{2}}{(1+i \Delta x)(2+i \Delta x)} \ln \left\{\frac{1+i \Delta x}{1+(i-q) \Delta x}\right\} \\
& +\frac{1}{1+i \Delta x} O(\Delta x)^{2} \\
< & \frac{\Delta x}{1+i \Delta x}-\frac{2 \Delta x}{2+i \Delta x} \operatorname{In}(1+q \Delta x)+\frac{1}{1+i \Delta x} O(\Delta x)^{2} \\
< & \frac{\Delta x}{1+i \Delta x}-\frac{2.5 q(\Delta x)^{2}}{(2+i \Delta x)(1+q \Delta x)} \\
< & \frac{(1.25+0.75 i \Delta x) \Delta x}{(1+i \Delta x)(2+i \Delta x)(1+q \Delta x)} \\
& \frac{3 \Delta x}{4(1+i \Delta x)(1+q \Delta x)} .
\end{aligned}
$$

QED

Now we can use the above lemmas to estimate $P(\gamma)$.

Lemma 7. There exists a constant $0<\eta<1$ which is independent of $N$ and $\Delta x$ such that the reflection potential of $\gamma$ emitting from the mesh point $(0,0)$ is less than $\eta\|\gamma\|$ when $\Delta x \ll 1$.

Proof. We calculate $P(\gamma)$ by two parts, denoted by $B_{1}\|\gamma\|$ and $B_{2}\|\gamma\|$ the corresponding total norms. $B_{1}\|\gamma\|$ denotes the reflection potential due to those $\left(R_{\gamma}^{1} \backslash R_{\gamma}^{3}\right)_{1}^{i}$, and $B_{2}\|\gamma\|$ denotes the reflection potential due to those $\left(R_{\gamma}^{1} \backslash R_{\gamma}^{3}\right)_{m}^{l}$ with $m \geqq 2$. Let $p \Delta x=0.9, q \Delta x=0.5$, we have

$$
B_{1}<\sum_{i=1}^{p} \frac{\Delta x}{1+i \Delta x}-\sum_{j=1}^{p-1} \sum_{i=1}^{j} \frac{(\Delta x)^{2}}{(1+i \Delta x)(1+(j-i+1) \Delta x)}=D_{1}
$$




$$
\begin{aligned}
B_{2}< & \sum_{j=1}^{q-2} \frac{\Delta x}{1+(j+1) \Delta x} \sum_{i=1}^{j} \frac{\Delta x}{(1+i \Delta x)(1+(j-i+1) \Delta x)} \\
& +\sum_{j=q-1}^{\infty} \frac{\Delta x}{1+(j+1) \Delta x} \sum_{i=1}^{q-1} \frac{\Delta x}{(1+i \Delta x)(1+(j-i+1) \Delta x)} \\
& +\frac{3}{4} \sum_{j=q}^{p-2} \frac{\Delta x}{1+(j+1) \Delta x} \sum_{i=q}^{j} \frac{\Delta x}{(1+i \Delta x)(1+(j-i+1) \Delta x)} \\
& +\frac{3}{4} \sum_{j=p-1}^{\infty} \frac{\Delta x}{1+(j+1) \Delta x} \sum_{i=q}^{p-1} \frac{\Delta x}{(1+i \Delta x)(1+(j-i+1) \Delta x)} \\
= & D_{2}, \quad<\frac{q x}{D_{2}}<\sum_{i=1}^{q} \frac{\Delta x}{1+i \Delta x}+\frac{3}{4} \sum_{i=q}^{p-1} \frac{\Delta x}{1+i \Delta x} \\
& <\ln (1+q \Delta x)+\frac{3}{4} \ln \left\{\frac{1+p \Delta x}{1+q \Delta x}\right\}+O(\Delta x)^{2} \\
& <0.583,
\end{aligned}
$$

when $\Delta x \ll 1$. Thus

$$
\begin{aligned}
B_{1}+B_{2} & <D_{1}+D_{2} \\
& <\ln 1.9+0.583-\sum_{j=1}^{p-1} \sum_{l=1}^{j} \frac{(\Delta x)^{2}}{(1+i \Delta x)(1+(j-i+1) \Delta x)} \\
& =\ln 1.9+0.583-J,
\end{aligned}
$$

where

$$
\begin{aligned}
J & >\sum_{i=2}^{p} \frac{2 \operatorname{In}\{1+(i-1) \Delta x\}}{2+i \Delta x}+O(\Delta x)^{2} \\
& >2 \int_{0}^{0.9} \ln \left(\frac{1+x}{2+x}\right) d x+O(\Delta x)^{2} .
\end{aligned}
$$

Thus when $\Delta x \ll 1$, we have

$$
\begin{aligned}
B_{1}+B_{2} & <\ln 1.9+0.583-0.23 \\
& =0.995,
\end{aligned}
$$

i.e. we can choose $\eta=0.995$.

Note 4. It's not important what exactly $B_{1}+B_{2}$ is. What is important is that $B_{1}+$ $B_{2}<\eta<1$.

Now we will give a technical estimation of the reflection potential for $\gamma$ emitting from the mesh point $(m, 0)$. 
Lemma 8. There exists a constant $\eta<1$ which is independent of $N, m$ and $\Delta x$ such that the reflection potential for a $2^{\text {nd }}$ hyperbolic wave $\gamma$ emitting from the mesh point $(m, 0)$ is also less than $\eta\|\gamma\|$ when $\Delta x \ll 1$.

Proof. Consider the mesh point $(m+n-l, n+l)$, where $0 \leqq l \leqq m$, we have

$$
\begin{aligned}
\left\|\left(R_{i}^{3}\right)_{m+n-l}^{n+l}\right\| /\|\gamma\|= & \sum_{j=0}^{n-1} \frac{\Delta x}{1+(m+j) \Delta x)} \\
& \times \sum_{i=0}^{l-1} \frac{(\Delta x)^{2}}{(1+(m+i+j-l) \Delta x)(1+(m+n+i-l) \Delta x)} \\
> & \sum_{j=0}^{n-1} \frac{(\Delta x)^{2}}{1+(m+j) \Delta x} \frac{1}{(n-j) \Delta x}\left\{\ln \left\{\frac{1+(m+j) \Delta x}{1+(m+j-l) \Delta x}\right\}\right. \\
& \left.-\ln \left\{\frac{1+(m+n) \Delta x}{1+(m+n-l) \Delta x}\right\}\right\}+\frac{1}{1+m \Delta x} O(\Delta x)^{2} .
\end{aligned}
$$

Now we need the following lemma.

Lemma 9. When $m$ and $n$ are fixed, $0<x<n \Delta x$,

$$
\mathscr{L}(x)=\frac{1+m \Delta x+x}{n \Delta x-x}\left\{\ln \left\{\frac{1+m \Delta x+x}{1+x}\right\}-\ln \left\{\frac{1+(m+n) \Delta x}{1+n \Delta x}\right\}\right\}
$$

is a decreasing function of $x$.

Proof.

$$
\begin{aligned}
\mathscr{L}^{\prime}(x)= & \frac{1+(n+m) \Delta x}{(n \Delta x-x)^{2}}\left\{\ln \left(\frac{1+m \Delta x+x}{1+x}\right)-\ln \left\{\frac{1+(m+n) \Delta x}{1+n \Delta x}\right\}\right\} \\
& -\frac{m \Delta x}{(n \Delta x-x)(1+x)} .
\end{aligned}
$$

Let

$$
\beta(x)=\ln \left(\frac{1+m \Delta x+x}{1+x}\right)-\ln \left\{\frac{1+(m+n) \Delta x}{1+n \Delta x}\right\}-\frac{m \Delta x(n \Delta x-x)}{(1+(m+n) \Delta x)(1+x)},
$$

then

$$
\beta^{\prime}(x)=\frac{(m \Delta x)^{2}(n \Delta x-x)}{(1+x)^{2}(1+(m+n) \Delta x)(1+m \Delta x+x)} .
$$

Since $0<x<n \Delta x$, then $\beta^{\prime}(x)>0$. Since $\lim _{x \rightarrow n \Delta x} \beta(x)=0$, then $\beta(x)<0$, i.e., $\mathscr{L}^{\prime}(x)<0$. QED 
Now we come back to the proof of Lemma 8. Since

$$
\begin{aligned}
& \lim _{\Delta x \rightarrow 0} \frac{1}{\Delta x}\left\{\ln \left\{\frac{1+(m+n-1) \Delta x}{1+(m+n-l-1) \Delta x}\right\}-\ln \left\{\frac{1+(m+n) \Delta x}{1+(m+n-l) \Delta x}\right\}\right\} \\
& =\frac{l \Delta x}{(1+(m+n) \Delta x)(1+(m+n-l) \Delta x)}
\end{aligned}
$$

then we have

$$
\begin{aligned}
& \left\|\left(R_{\gamma}^{3}\right)_{m+n-l}^{n+l}\right\| /\|\gamma\| \\
& \geqq \frac{l \Delta x(1+(m+n-1) \Delta x)}{(1+(m+n) \Delta x)(1+(m+n-l) \Delta x)} \times \sum_{i=0}^{n-1} \frac{(\Delta x)^{2}}{(1+(m+i) \Delta x)^{2}} \\
& >\frac{\ln (\Delta x)^{3}}{(1+m \Delta x)(1+(m+n) \Delta x)(1+(m+n-l) \Delta x)}+\frac{1}{1+m \Delta x} O(\Delta x)^{2} .
\end{aligned}
$$

If we choose $N(\Delta x)^{2} \leqq(\Delta x)^{\frac{1}{2}}$, since $n<N$ we have

$$
\begin{aligned}
\|\left(R_{\gamma}^{1} \backslash\right. & \left.R_{\gamma}^{3}\right)_{n}^{m+n} \| \\
= & \frac{\Delta x}{1+(m+n) \Delta x}\|\gamma\|-\left(R_{\gamma}^{3}\right)_{n}^{m+n} \\
\leqq & \left\{\frac{\Delta x}{1+(m+n) \Delta x}-\frac{n m(\Delta x)^{3}}{(1+m \Delta x)(1+(m+n) \Delta x)(1+n \Delta x)}\right. \\
& \left.+\frac{1}{1+m \Delta x} O(\Delta x)^{2}\right\}\|\gamma\| \\
= & \left\{\frac{\Delta x+O(\Delta x)^{\frac{3}{2}}}{(1+m \Delta x)(1+n \Delta x)}\right\}\|\gamma\|, \\
\left\|\left(R_{\gamma}^{1} \backslash R_{\gamma}^{3}\right)_{m+n-l}^{n+l}\right\| & \leqq \\
\leqq & \left\{\frac{\Delta x}{1+(m+n) \Delta x}-\frac{1}{(1+m \Delta x)(1+(m+n) \Delta x)(1+(m+n-l) \Delta x)}\right. \\
& \left.+\frac{1}{1+m \Delta x} O(\Delta x)^{2}\right\}\|\gamma\| \\
= & \left\{\frac{(1+(m-l) \Delta x) \Delta x}{(1+m \Delta x)(1+(m+n-l) \Delta x)}+\frac{1}{1+m \Delta x} O(\Delta x)^{2}\right\}\|\gamma\|
\end{aligned}
$$


We estimate the reflection potential $P(\gamma)$ by two parts, denoted by $P_{\Omega_{l}}(\gamma)$, $i=1,2$, where

$$
\begin{aligned}
\Omega_{1} \equiv & \{(x, t): 0 \leqq t \leqq m \Delta t \text { and } t \geqq m \Delta t \\
& \left.1+\left[\frac{t-m \Delta t}{\Delta t}\right] \Delta x \leqq x \leqq 1+\left(m+\left[\frac{t}{\Delta t}\right]\right) \Delta x\right\}, \\
\Omega_{2} \equiv & \left\{(x, t): t \geqq m \Delta t, 1 \leqq x \leqq 1+\left[\frac{t-m \Delta t}{\Delta t}\right] \Delta x\right\}
\end{aligned}
$$

By (17) and $\frac{1}{(1+a)(1+b)}<\frac{1}{1+(a+b)}$ for $a, b>0$, it is easy to prove that the reflection potential in $\Omega_{2}$ due to $\gamma$ is less than the reflection potential of a $2^{\text {nd }}$ hyperbolic wave $\bar{\gamma}$ emitted from the mesh point $(0, m)$ with the norm being $\left(\frac{1+O(\Delta x)^{\frac{1}{2}}}{1+m \Delta x}\right)\|\gamma\|$. Since all the lemmas are true for arbitrary time, by Lemma 7 we have

$$
P_{\Omega_{2}}(\gamma) \leqq\left\{\frac{\eta\left(1+O(\Delta x)^{\frac{1}{2}}\right)}{1+m \Delta x}\right\}\|\gamma\| .
$$

By (18) we have

$$
\begin{aligned}
P_{\Omega_{1}}(\gamma) & <\sum_{l=0}^{m} \sum_{n=0}^{N}\left\{\frac{(1+(m-l) \Delta x)(\Delta x)^{2}}{(1+m \Delta x)(1+(m+n-l) \Delta x)^{2}}+\frac{1}{1+m \Delta x} O(\Delta x)^{3}\right\}\|\gamma\| \\
& <\left\{\sum_{l=0}^{m} \sum_{n=0}^{\infty} \frac{(1+(m-l) \Delta x)(\Delta x)^{2}}{(1+m \Delta x)(1+(m+n-l) \Delta x)^{2}}+\sum_{l=0}^{m} \frac{1}{1+m \Delta x} O(\Delta x)^{\frac{3}{2}}\right\}\|\gamma\| \\
& =\left\{\sum_{l=0}^{m} \frac{\Delta x}{1+m \Delta x}+O(\Delta x)^{\frac{1}{2}}\right\}\|\gamma\| \\
& =\left\{\frac{m \Delta x}{1+m \Delta x}+O(\Delta x)^{\frac{1}{2}}\right\}\|\gamma\| .
\end{aligned}
$$

But (19) and (20) are not sufficient to prove that the reflection potential is less than $\eta\|\gamma\|$ for some fixed $\eta<1$. Because the constant in front of $\|\gamma\|$ in (20) will tend to 1 as $m$ tends to infinity. In order to get a $\eta<1$ which is independent of $N, m$ and $\Delta x$, we have to refine the estimate of $P_{\Omega_{1}}(\gamma)$. Now we need the following lemmas.

Lemma 10. For $l \geqq \frac{4}{5} m \Delta x, m \Delta x \geqq 100, \Delta x \ll 1$, there exists constants $D$ and $\eta_{1}>0$ which are independent of $N, m$ and $\Delta x$, such that if $w \geqq D m, 0 \leqq n \leqq m$,

$$
\begin{aligned}
& \frac{1+(m+n) \Delta x}{1+(w-n) \Delta x}\left\{\ln \left\{\frac{1+(m+n) \Delta x}{1+(m+n-l) \Delta x}\right\}-\ln \left\{\frac{1+(m+w) \Delta x}{1+(m+w-l) \Delta x}\right\}\right\} \\
& >\left(1+\eta_{1}\right) \frac{l \Delta x}{1+(m+w-l) \Delta x} .
\end{aligned}
$$


Lemma 11. There exists a constant $\eta_{2}>0$ such that if $l \geqq\left(1-\eta_{2}\right) m, n \geqq$ $D m, m \Delta x \geqq 100$ and $\Delta x \ll 1$, we have

$$
\left\|\left(R_{\gamma}^{1} \backslash R_{\gamma}^{3}\right)_{m+n-l}^{n+l}\right\| \equiv 0 .
$$

The proofs for Lemma 10 and 11 are omitted. Thus when $m \Delta x \geqq 100$, we have

$$
\begin{aligned}
& P_{\Omega_{1}}(\gamma) \\
& \quad \leqq\left\{\frac{m \Delta x}{1+m \Delta x}-\sum_{w=D m}^{\infty} \sum_{l=\left(1-\eta_{2}\right) m}^{m} \frac{1+(m-l) \Delta x}{(1+m \Delta x)(1+(m+w-l) \Delta x)^{2}}+O(\Delta x)^{\frac{1}{2}}\right\}\|\gamma\| \\
& \quad \leqq\left\{\frac{m \Delta x}{1+m \Delta x}-\eta_{3}+O(\Delta x)^{\frac{1}{2}}\right\}\|\gamma\| .
\end{aligned}
$$

where $\eta_{3}>0$ is a constant independent of $N, m$ and $\Delta x$. By (19) and (22), we know that when $\Delta x \ll 1$, there exists a constant $\eta<1$ such that

$$
P(\gamma)=P_{\Omega_{1}}(\gamma)+P_{\Omega_{2}}(\gamma) \leqq \eta\|\gamma\|,
$$

where $\eta$ is independent of $N, m$ and $\Delta x$. QED

Now we can conclude the proof of Lemma 3. Before doing this, we give the following definitions.

Definition 11. For any hyperbolic wave $\gamma$ of the $2^{\text {nd }}$ family emitting from the mesh point $(m, 0)$, we define

$$
U_{m}^{i}\left(R_{\gamma}^{i} \backslash R_{\gamma}^{i+2}\right)=\sum_{\bar{m}}\left\|\left(R_{\gamma}^{l} \backslash R_{\gamma}^{i+2}\right)_{m}^{N}\right\|
$$

and

$$
V_{m}^{J}\left(R_{\gamma}^{2 j-1} \backslash R_{\gamma}^{2 J+1}\right)=\sum_{i}\left\|\left(R_{\gamma}^{2 j-1} \backslash R_{\gamma}^{2 j+1}\right)_{1}^{i}\right\|
$$

Let

$$
\begin{aligned}
\theta^{i} & =\sup _{m}\left\{U_{m}^{i}\left(R_{\gamma}^{i} \backslash R_{\gamma}^{l+2}\right) /\|\gamma\|\right\}, \\
\Theta^{j} & =\sup _{m}\left\{V_{m}^{j}\left(R_{\gamma}^{2 j-1} \backslash R_{\gamma}^{2 J+1}\right) /\|\gamma\|\right\},
\end{aligned}
$$

and

$$
\hat{\eta}=\sup \{P(\gamma) /\|\gamma\| \mid \text { for all } \gamma \text { on } t=0,\|\gamma\| \neq 0\} \text {. }
$$

By the above discussion, we know that $\hat{\eta}\|\gamma\|$ is a uniform upper bound for $P(\gamma)$, where $\gamma$ is any hyperbolic wave of the $2^{\text {nd }}$ family in the simplified scheme and in the region $0 \leqq t \leqq N \Delta t, x \geqq 1$. And it is easy to prove the following lemma.

Lemma 12. There exists a constant $0<\zeta<1$ which is independent of $N, m$ and $\Delta x$, such that

$$
\theta^{1}, \Theta^{1} \leqq \ln 2+\zeta, \quad \text { when } \Delta x \ll 1
$$

Proof of Lemma 3. Since $U_{m}^{i}\left(R_{\gamma}^{i} \backslash R_{\gamma}^{i+2}\right)$ and $V_{m}^{j}\left(R_{\gamma}^{2 j-1} \backslash R_{\gamma}^{2 j+1}\right)$ will approach zero when $i$ approaches $l, j$ approaches $\left[\frac{l}{2}\right]$ and $\Delta x$ approaches 0 , where $l$ is defined by 
$R_{\gamma}^{l+1} \equiv \phi$, where $\phi$ is the empty set. Then for any hyperbolic wave $\gamma$ of the $2^{\text {nd }}$ family emitting from the mesh point $(m, 0)$, we have

$$
R T^{1}(\gamma)=\sum_{l=\text { odd }, m>0}\left\|\left(R_{\gamma}^{i} \backslash R_{\gamma}^{i+2}\right)_{m}^{N}\right\| \leqq \sum_{i=\text { odd }} \theta^{l}\|\beta\|,
$$

and

$$
R T^{2}(\gamma) \leqq \sum_{l=\text { even }} \theta^{l}\|\gamma\|
$$

By the reflection potential estimate in the last section, we have

$$
\theta^{l+2}<\hat{\eta} \theta^{l}, \quad \Theta^{l+2}<\hat{\eta} \Theta^{l}, \quad i>0
$$

Thus we have

$$
\begin{aligned}
R T^{1}(\gamma) & <(\ln 2+\zeta)\left\{\sum_{i=0}^{\infty}(\hat{\eta})^{i}\right\}\|\gamma\| \\
& <\frac{\ln 2+\zeta}{1-\hat{\eta}}\|\gamma\| .
\end{aligned}
$$

Since $U_{m}^{2}\left(R_{\gamma}^{2} \backslash R_{\gamma}^{4}\right)$ is equal to the reflection potential of $\gamma$, then

$$
R T^{2}(\gamma)<\frac{\hat{\eta}}{1-\hat{\eta}}\|\gamma\| .
$$

Since the total norm of the reflections on $x=1,0 \leqq t \leqq N k$ is equal to the total norm of the reflections approaching $x=1,0 \leqq t \leqq N k$, then

$$
R T^{0}(\gamma) \leqq \sum_{j=1}^{\left[\frac{1}{2}\right]+1} \Theta^{J}\|\gamma\|<\frac{\ln 2+\zeta}{1-\hat{\eta}}\|\gamma\|
$$

If $\gamma$ is a hyperbolic wave of the $1^{\text {st }}$ family emitting from the mesh point $(m, 0)$. When $\Delta x \ll 0$, it is straightforward to prove that

$$
\begin{aligned}
R T^{1}(\gamma) & <\{1+\ln (1+m \Delta x)+O(\Delta x)\} \frac{\ln 2+\zeta}{1-\hat{\eta}}\|\gamma\| \\
& <\{2+\ln (1+m \Delta x)\} \frac{\ln 2+\zeta}{1-\hat{\eta}}\|\gamma\|, \\
R T^{2}(\gamma) & <\{2+\ln (1+m \Delta x)\} \frac{\hat{\eta}}{1-\hat{\eta}}\|\gamma\|, \\
R T^{0}(\gamma) & <\{2+\ln (1+m \Delta x)\} \frac{\ln 2+\zeta}{1-\hat{\eta}}\|\gamma\| .
\end{aligned}
$$

By (23) to (28), Lemma 3 is proved by choosing $\bar{G}=\frac{2}{1-\hat{\eta}}$.

Now we can derive the formula relating the hyperbolic waves on $t=N \Delta t$ and those on $t=0$. Before stating the theorem in this section, we introduce some notations: For our reduced model problem, we use $\gamma_{m, l}^{j}$ to denote the hyperbolic wave of the $j^{\text {th }}$ family on the mesh point $(m, i)$ in the simplified scheme, where $j=1,2$. 
Theorem 4. For the waves evolution in the simplified scheme, if $\Delta x \ll 1$, there exists a constant $G_{1}$ which is independent of $N$ and $\Delta x$, such that

$$
\begin{gathered}
\sum_{j, m}\left\|\gamma_{m, N}^{j}\right\| \leqq G_{1} \sum_{m}\left\{\{2+\ln (1+m \Delta x)\}\left\|\gamma_{m, 0}^{1}\right\|+\left\|\gamma_{m, 0}^{2}\right\|\right\}, \\
\sum_{i>0}\left\|\gamma_{0, i}^{2}\right\| \leqq G_{1} \sum_{m}\left\{\{2+\ln (1+m \Delta x)\}\left\|\gamma_{m, 0}^{1}\right\|+\left\|\gamma_{m, 0}^{2}\right\|\right\},
\end{gathered}
$$

where the sum are taken over all possible $m, j$ and $i$.

Proof. By the simplified scheme, we have

$$
\begin{aligned}
\sum_{j, m}\left\|\gamma_{m, N}^{j}\right\| & \leqq \sum_{j, m}\left\|\gamma_{m, 0}^{j}\right\|+\sum_{j, m, k}\left\|\sum_{i}\left(R_{\gamma_{m, 0}^{l}}^{j}\right)_{k}^{N}\right\| \\
& \leqq \sum_{j, m}\left\|\gamma_{m, 0}^{j}\right\|+\sum_{j, m} \sum_{i, k}\left\|\left(R_{\gamma_{m, 0}^{i}, \backslash}^{j} \backslash R_{\gamma_{m, 0}^{j}}^{l+2}\right)_{k}^{N}\right\| \\
& \leqq \sum_{j, m}\left\|\gamma_{m, 0}^{j}\right\|+\sum_{j, m}\left\{R T^{1}\left(\gamma_{m, 0}^{j}\right)+R T^{2}\left(\gamma_{m, 0}^{j}\right)\right\} \\
& \leqq(2 \bar{G}+1) \sum_{m}\left\{\{2+\ln (1+m \Delta x)\}\left\|\gamma_{m, 0}^{1}\right\|+\left\|\gamma_{m, 0}^{2}\right\|\right\} .
\end{aligned}
$$

Similarly, we have

$$
\begin{aligned}
\sum_{l>0}\left\|\gamma_{0, l}^{2}\right\| & \leqq \sum_{m}\left\|\gamma_{m, 0}^{1}\right\|+\sum_{j, m, i}\left\|\sum_{l}\left(R_{\gamma_{m, 0}^{l},}^{l}\right)_{0}^{l}\right\| \\
& \leqq \sum_{m}\left\|\gamma_{m, 0}^{1}\right\|+\sum_{j, m} \sum_{l, i}\left\|\left(R_{\gamma_{m, 0}^{l}}^{l} \backslash R_{\gamma_{m, 0}^{l}}^{l+2}\right)_{0}^{i}\right\| \\
& \leqq \sum_{m}\left\|\gamma_{m, 0}^{1}\right\|+\sum_{j, m} R T^{0}\left(\gamma_{m, 0}^{j}\right) \\
& \leqq(\bar{G}+1) \sum_{m}\left\{\{2+\ln (1+m \Delta x)\}\left\|\gamma_{m, 0}^{1}\right\|+\left\|\gamma_{m, 0}^{2}\right\|\right\}
\end{aligned}
$$

Let $G_{1}=2 \bar{G}+1$, we get (29) and (30).QED

\section{Proof of Theorem 1}

Now we can conclude the proof for Theorem 1 . That is, we will prove that there exists a uniform bound for the $L_{\infty}$ norm and the total variation norm of $(u(x, t), \rho(x, t))$ for and time, where $(u(x, t), \rho(x, t))$ is the solution for the model problem.

Proof of Theorem 1. As shown in Sect. 2, the model problem in Theorem 1 can be reduced to the ideal model problem discussed in Sect. 7. Thus by Theorem 4 and Lemma 2 , if $\Delta x \ll 1$, we have

$$
\begin{aligned}
\sum_{j, m}\left\|\gamma_{m, N}^{j}\right\| & \leqq G_{2} \delta, \quad \sum_{l}\left\|\gamma_{0, l}^{2}\right\| \leqq G_{2} \delta, \\
|\rho(1, T)-\bar{\rho}| & <G_{2} \delta,
\end{aligned}
$$

where $G_{2}$ is a constant independent of $N, \Delta x$ and $T=N \Delta t$. 
By the definition of standing waves, we know that $\rho$ is constant and $|u|$ is decreasing while crossing a standing wave from left to right in the ideal model problem. Since $u(1, t) \equiv 0$, the strengths of the rebounded waves of $x=1$ are equal to those of the impinging waves. Thus we have

$$
|x u(x, T)|<G_{3} \delta, \quad|\rho(x, T)-\bar{\rho}|<G_{3} \delta, \quad x \geqq 1,
$$

where $G_{3}$ is constant independent of $N$ and $\Delta x$.

Furthermore, if a left state $\left(\rho_{l}, u_{l}, a_{l}\right)$ and a right state $\left(\rho_{r}, u_{r}, a_{r}\right)$ are connected by only a standing wave $\gamma^{0}$ in the solutions of the Riemann problem, where $a_{l}=x_{0}^{2}$ and $a_{r}=\left(x_{0}+\Delta x\right)^{2}$, the Glimm's strength of $\gamma^{0}$ satisfies

$$
\left|\gamma^{0}\right|=\frac{2 \Delta x}{x_{0}^{2}}\left|x_{0} u\right| \leqq \frac{2 G_{3} \delta \Delta x}{x_{0}^{2}}
$$

Since the $L_{1}$ norm of $\frac{1}{x^{2}}$ is bounded when $x \in[1, \infty)$, we have

$$
\operatorname{Var}\{u(x, t)\}, \quad \operatorname{Var}\{\rho(x, t)\}<G \delta, \quad x \geqq 1,
$$

where $G$ is a constant independent of $N$ and $\Delta x$. Since $N$ can be arbitrarily large, then the bounds obtained above are uniform with respect to any time. And the proof for Theorem 1 is completed. QED

Acknowledgement. This paper is my Ph.D thesis under the supervision of Professor J. Blake Temple. I am very grateful to Blake for sharing his ideas and a guidance and encouragement during my Ph.D study, especially at every stage of this work.

\section{References}

1. Glimm, J.: Solutions in the large for nonlinear hyperbolic systems of equations. Comm. Pure Appl. Math. 18, 697-715 (1965)

2. Lax, P.D.: Hyperbolic systems of conservation laws and the mathematical theory of shock waves. SIAM Reg. Conf. Lecture 11, Philadelphia (1973)

3. Kato, T.: The Cauchy problem for quasilinear symmetric hyperbolic systems. Arch. Rational Mech. Anal. 58, 181-205 (1975)

4. Makino, T., Mizohata, K., Ukai, S.: The global weak solutions of the copressible Euler equation with spherical symmetry. To appear

5. Liu, T.P.: Nonlinear resonance for quasilinear hyperbolic equation. J. Math. Phys. 28(11), 2593-2602 (1987)

6. Liu, T.P.: Quasilinear hyperbolic systems. Commun. Math. Phys. 68, 141-172 (1979)

7. Marchesin, D., Paes-leme, P.J.: A Riemann problem in gas dynamics with bifurcaton, PUC Report, MAT 02/84, 1984

8. Nishida, T., Smoller, J.: Mixed problems for nonlinear coservation laws. J. Diff. Equ. 23, 244-267 (1977)

9. Nishida, T., Smoller, J.: Solutions in the large for some nonlinear hyperbolic conservation laws. Comm. Pure Appl. Math. 26, 183-200 (1973)

10. DiPerna, R.: Convergence of the viscosity method for isentropic gas dynamics. Commun. Math. Phys. 91, 1-30 (1983)

11. Temple, B.: Decay with a rate for noncompactly supported solutions of conservation laws. Tran. Am. Math. Soc. 298, no.1, 43-82 (1986)

12. Temple, B., Goodman: Private talks and notes

13. Hunter, J.: Private talks 